\title{
The Valuation of American Barrier Options Using the Decomposition Technique ${ }^{1}$
}

\author{
Bin $\mathrm{Gao}^{2}$ \\ Jing-zhi Huang ${ }^{3}$ \\ Marti Subrahmanyam ${ }^{4}$
}

First draft: October 29, 1995

This draft: October 25, 1999

\footnotetext{
${ }^{1}$ This is a substantially revised version of an earlier paper circulated under the title "Analytical Approach to the Valuation of American Path-Dependent Options." We acknowledge comments and suggestions from two anonymous referees that were extremely useful in revising earlier drafts of this paper. We also acknowledge the extensive and detailed comments of the editor, Michael Selby, that helped to improve the paper substantially, in terms of both content and exposition. We thank James Bodurtha, Jr., Young-Ho Eom, Nengjiu Ju, A.R. Radhakrishnan, Rangarajan Sundaram, and especially Sanjiv Das for comments and discussions, and participants at the Courant Institute Mathematical Finance Seminar (March 1996), the First Annual Computational Finance Conference at Stanford University (August 1996), and the Cornell-Queen's Conference on Derivatives (May 1997) for comments on earlier drafts of the paper.

${ }^{2}$ Kenan-Flagler Business School, University of North Carolina, Chapel Hill, NC 27599. Tel: (919) 962-7182. Fax: (919) 962-2068. E-mail: gaob@icarus.bschool.unc.edu

${ }^{3}$ Smeal College of Business, Penn State University, University Park, PA 16802. Tel: (814) 8633566. Fax: (814) 865-3362. E-mail: jxh56@psu.edu

${ }^{4}$ Stern School of Business, New York University, New York, NY 10012. Tel: (212) 998-0348. Fax: (212) 995-4233. E-mail: msubrahm@stern.nyu.edu.
} 


\begin{abstract}
In this paper, we propose an alternative approach for pricing and hedging American barrier options. Specifically, we obtain an analytic representation for the value and hedge parameters of barrier options, using the decomposition technique of separating the European option value from the early exercise premium. This allows us to identify some new put-call "symmetry" relations and the homogeneity in price parameters of the optimal exercise boundary. These properties can be utilized to increase the computational efficiency of our method in pricing and hedging American options.

Our implementation of the obtained solution indicates that the proposed approach is both efficient and accurate in computing option values and option hedge parameters. Our numerical results also demonstrate that the approach dominates the existing lattice methods in both accuracy and efficiency. In particular, the method is free of the difficulty that existing numerical methods have in dealing with spot prices in the proximity of the barrier, the case where the barrier options are most problematic.
\end{abstract}




\section{Introduction}

Non-standard or exotic options are widely used today by banks, corporations and institutional investors, in their management of risk. The main reason for their popularity is that although standard put and call options are useful risk management tools, they may not be suitable for hedging certain types of risks. For instance, a corporation may wish to control its raw material costs by limiting the average price paid for a commodity over time (Asian options), or obtaining protection, contingent upon the price breaching a barrier (barrier options). In these and other situations, the use of standard options may involve over-hedging (i.e. providing protection against risks that need not be hedged), and hence higher costs. Consequently, the use of non-standard options may not only fit the risk to be hedged better, but also lower the hedging cost, in such cases.

Although the payoff functions of non-standard options are often not more complex than that of standard options, this is not true for the pricing and hedging of such options. In most cases, such as Asian, barrier and look-back options, whose payoffs are path-dependent, closed-form solutions are hard to come by. This is true even for European-style contracts, except for the special case where the underlying asset price follows a geometric Brownian motion. Therefore, numerical schemes have to be used to calculate the option prices and hedge parameters for American-style options and even for some European-style options.

The focus of this paper is on the valuation of barrier options. Barrier options are options that are either extinguished ("out") or established ("in"), when the price of the underlying asset crosses a particular level ("barrier"). Common examples are "down-and-out," "downand-in," "up-and-out" and "up-and-in" options, both calls and puts. An additional feature of some barrier options is that a rebate is paid when the option is extinguished or an additional premium is due when the option is established. Barrier options are among the most common exotic options that are used in the foreign exchange, interest rate and equity options markets. ${ }^{1}$ They are used by hedgers to obtain insurance protection above or below particular levels of the price of the underlying asset. They are also used by speculators, who have a directional view, to obtain a somewhat less expensive directional play on an underlying asset. In some instances, barrier options are American-style. Barrier options also include "capped" options as special cases.

Common approaches to option valuation and hedging such as lattice and simulation methods can be problematic when applied to barrier options. It is known that for such options, the binomial method is subject to severe convergence problems, and consequently, can lead to huge errors even with a large number of time-steps. The reason is that the payoff of a barrier option is very sensitive to the position of the barrier in the lattice - a "knockout" option behaves very much like a standard option when the underlying asset

\footnotetext{
${ }^{1} \mathrm{~A}$ recent estimate cited by $\mathrm{Hsu}(1997)$ computes the size of the barrier options market to be over 2 trillion dollars in 1996. The market has grown considerably since that time.
} 
price is far away from the barrier, but has a near-zero "expected" payoff, when it is close to the barrier.

Boyle and Lau (1994) and Ritchken (1995) develop a restricted binomial/trinomial method to overcome the problem. However, with these methods, it is still extremely difficult to achieve convergence when the barrier is close to the current price of the underlying asset (the "near-barrier" problem). Gao (1996) proposes an "adaptive mesh" method, which overcomes some of the problems posed by the above models. Even with this modification, the computational time increases as the current underlying price gets closer to the barrier, although at a much slower pace. Further, as shown by Gao, the computational intensity of lattice methods is proportional to the maturity and the square of the volatility. Consequently, the computational costs associated with pricing long maturity and high volatility contracts can be prohibitively high. Cheuk and Vorst (1996) show that a trinomial lattice with a flexible drift can alleviate the "near-barrier" problem. However, the method permits probabilities to become negative, and can produce fairly large pricing errors for long-term contracts when volatility is high and the spot price is close to the barrier. ${ }^{2}$

In this paper, we propose a quasi-analytic approach to the valuation of American barrier options. Specifically, we obtain an analytic representation for the value and hedge parameters of barrier options using the decomposition technique. Under this representation, the price of an American-style barrier option can be split up into the price of a standard European barrier option and an early exercise premium. Similar results can also be obtained for hedge parameters. By using the put-call "symmetry" condition that we derive, and the well-known relationship between "up-and-out" and "up-and-in" options, we can extend our results to a whole series of barrier options. We also identify some characteristics of the optimal exercise boundary: homogeneity in the strike and barrier prices, translational invariance in time, and monotonicity in time, and monotonicity in the strike and barrier prices. As mentioned later on, these properties are important in the practical implementation of the method we propose, since the boundary does not have to be recomputed separately for each option.

Our method of implementing the analytic representation using the decomposition technique allows us to calculate both option prices and hedge parameters efficiently and accurately. For example, in the case of American "up-and-out" options, our numerical results indicate that the approach outperforms both the Ritchken (1995) method and the Cheuk and Vorst (1996) model. In particular, the method we propose is faster than the Ritchken method by two orders of magnitude for equally accurate prices and hedge ratios, when the underlying asset price is close to the barrier. Moreover, in contrast with the other methods, the computational time required by the analytic approach hardly increases as the current

\footnotetext{
${ }^{2}$ In a recent paper, Rogers and Stapleton (1998) provide an alternative lattice based method for the valuation of barrier options, in which the number of time steps taken is random. However, they implement their method only for the case of European barrier options and standard American options.
} 
underlying asset price gets closer to the barrier. In fact, this "near-barrier" problem, which is endemic in the lattice methods, is completely eliminated in our formulation. This is because the optimal exercise boundary, the sufficient input function of our valuation formula, is independent of the current underlying price. The method proposed here also applies to "capped" options and might be extended to other types of path-dependent options, such as Asian options, whose payoff functions have a Markovian representation in the state space of low dimensionality. ${ }^{3}$

The paper is organized as follows. In Section 2, an analytic representation is derived first for the option price and hedge parameters under the assumption that the underlying asset price process follows a geometric Brownian motion. Put-call "symmetry" conditions and some properties of the optimal exercise boundary are then identified that extend the analytic results to a whole range of related barrier options. Section 3 discusses the implementation of the quasi-analytic formulae and presents our numerical results. Section 4 concludes the paper.

\section{$2 \quad$ A Pricing Model}

In this section, we first obtain an analytic representation for the price of American barrier options using the decomposition technique. Based on this representation, we then derive some properties of the optimal exercise boundary.

The basic idea of the decomposition technique, proposed by MacMillan (1986) and Barone-Adesi and Whaley (1987), is to divide the price of an American option into that of a similar European option and the early exercise premium. This approach was further developed and specific results were obtained for the case of the log-normal underlying price process by Kim (1990), Jacka (1991), Carr, Jarrow and Myneni (1992), and Ho, Stapleton and Subrahmanyam (1997a). Specifically, an American option can be considered as a sum of two sets of cash flows using the decomposition approach: the value of the terminal cash flow at expiration and the value of the intermediate cash flows between the valuation date and expiration date. ${ }^{4}$ The former represents the value of an otherwise identical European option, and the latter, the value of the exercise privilege associated with an American option. Under the risk-neutral pricing framework, the value of an American option is equal to the sum of the expectation of these cash flows discounted by the risk-free rate.

Before proceeding with the analysis, we first define our notation as follows:

\footnotetext{
${ }^{3}$ Hansen and Jorgensen (1997) apply the method to floating-strike Asian options.

${ }^{4}$ See also Geske and Johnson (1984), Selby and Hodges (1987), and Schroder (1989).
} 
$c: \quad$ the price of a standard European call option.

$C: \quad$ the price of a standard American call option.

$c_{j}$ : the price of a non-standard European call option of type $j$. e.g., " $j=$ uo" denotes an "up-and-out" barrier option.

$C_{j}$ : the price of a non-standard American call option of type $j$.

$p: \quad$ the price of a standard European put option.

$P: \quad$ the price of a standard American put option.

$p_{j}: \quad$ the price of a non-standard European put option of type $j$.

$P_{j}: \quad$ the price of a non-standard American put option of type $j$.

We also use a superscript "o" to denote standard options. For instance, $C^{o}$ and $c^{o}$ represents the price of a standard American option and a standard European call option respectively. A superscript " $p$ " denotes the American premium due to the early exercise feature. We also make some assumptions that are common in the option pricing literature as follows:

Assumption 1 The capital market is complete and perfect. Trading takes place continuously and without transaction costs.

Assumption 1 allows us to use the risk-neutral pricing framework proposed by Cox and Ross (1976), and formalized and extended by Harrison and Kreps (1979), and Harrison and Pliska (1981). In the analysis that follows, we work under the risk-neutral measure.

Assumption 2 There are two tradeable assets in the market, a risky asset and a riskless asset. The continuously compounded interest rate $r$ is constant. ${ }^{5}$ The risky asset pays a constant dividend yield of $\delta \geq 0$, and its price process $\left\{S_{t} ; t \geq 0\right\}$ follows a geometric Brownian motion. ${ }^{6}$ Namely,

$$
d S_{t}=S_{t}(r-\delta) d t+\sigma S_{t} d W_{t}
$$

where $\delta$ and $\sigma$ are constants, and $W$ is a one-dimensional standard Brownian motion.

As shown later on, one advantage of making this assumption is that we can obtain an explicit expression for the early exercise premium, and as a result, a quasi-analytic solution for the price of an American barrier option, for instance, an "up-and-out" put option.

\footnotetext{
${ }^{5}$ The analysis can be extended to the case of a time-varying (deterministic) interest rate and dividend yield. In principle, the effect of stochastic interest rates can also be incorporated into the analysis along the lines proposed by Ho, Stapleton, and Subrahmanyam (1997a), although the details of the implementation are likely to be complex.

${ }^{6}$ The available empirical evidence suggests that that this assumption may not always be a good one. Nonetheless, the log-normal case can serve as a benchmark, since the Black-Scholes (1973) model, which is based on this assumption, is widely used and understood in practice. The analysis presented here can be extended to the case of time-varying (deterministic) volatility. However, the case of stochastic volatility would involve additional complexities, as in the case of standard options.
} 
Consequently, we can perform comparative statics analysis and examine analytically the properties of the optimal exercise boundary. We can also derive a put-call "symmetry" relation which allows us to extend the pricing models to a whole set of barrier options.

Without loss of generality, we consider an American-style "up-and-out" put option on the risky asset with a strike price $K$, a barrier $H$, maturity $T$, and a payoff $h\left(S_{t}\right)=$ $\left(K-S_{t}\right)^{+}$. The non-standard feature here is that if the asset price "hits" a barrier, the option becomes worthless. (Unless otherwise stated, a zero rebate is assumed throughout the paper, for simplicity. It is relatively easy to relax this assumption.)

Two cases are worth analyzing here: a) $H>K$ [out-of-the-money "up-and-out"]; b) $H \leq K$ [in-the-money (at-the-money) "up-and-out"]. Note that in the terminology of barrier options "in-the-money" or "out-of-the-money" are not related to the usual definition where $S<K$ or $S>K$.

\subsection{Out-of-the-Money "Up-and-Out" Puts}

We consider out-of-the-money American "up-and-out" put options, case a) above, in this subsection. Assume there exists an option pricing function s.t. $G: \mathcal{R}_{++} \times[0, T] \rightarrow \mathcal{R}_{+}$. Define the continuation region, $\mathcal{C}$, in which early exercise is not optimal, and the stopping region, $\mathcal{S}$, in which it is, as follows:

$$
\begin{aligned}
& \mathcal{C}=\left\{\left(S_{t}, t\right) \mid G\left(S_{t}, t\right)>h\left(S_{t}\right)\right\} \\
& \mathcal{S}=\left\{\left(S_{t}, t\right) \mid G\left(S_{t}, t\right) \leq h\left(S_{t}\right)\right\}
\end{aligned}
$$

As demonstrated in McKean (1965) and van Moerbeke (1976), the American option problem can be converted into a free-boundary problem. Under this formalization, $G\left(S_{t}, t\right)$ is the solution to the following problem [see Duffie (1992, p. 125) for details on the regularity conditions on functions $G$ and $h]$ :

$$
\begin{array}{rlrl}
\left(\mathcal{D}^{s}-r\right) G\left(S_{t}, t\right) & =0 & & \forall(S, t) \in \mathcal{C} \\
G\left(S_{T}, T\right) & =h_{T} & & \\
G\left(S_{t}, t\right) & >h_{t} & & \forall(S, t) \in \mathcal{C} \\
G\left(S_{t}, t\right) & \left.=h_{t}, t\right) \in \mathcal{S} \\
\frac{\partial}{\partial S_{t}} G\left(S_{t}, t\right) & =\frac{\partial}{\partial S_{t}} h_{t} & & \forall(S, t) \in \partial \mathcal{C}
\end{array}
$$

where the operator $\mathcal{D}^{s}$ is defined as follows

$$
\mathcal{D}^{s}=\frac{\partial}{\partial t}+(r-\delta) S \frac{\partial}{\partial S}+\frac{\sigma^{2} S^{2}}{2} \frac{\partial^{2}}{\partial S^{2}}
$$

Note that the option will be knocked out at $t$ if $M_{0}^{t} \geq H$, where $M_{t_{1}}^{t_{2}} \equiv \sup _{t_{1} \leq \tau \leq t_{2}} S_{\tau}$. 
Theorem 1 Consider an American-style "up-and-out" put option with $H>K$, whose payoff upon exercise is $h\left(S_{t}\right)=\left(K-S_{t}\right)^{+} \forall t \in[0, T]$. The price of the option is given by

$$
G\left(S_{0}, 0\right)=E\left[e^{-r T}\left(K-S_{T}\right)^{+} I_{\left\{M_{0}^{T}<H\right\}}\right]+\int_{0}^{T} e^{-r t} E\left[\left(r K-\delta S_{t}\right) I_{\left\{M_{0}^{t}<H ;\left(S_{t}, t\right) \in \mathcal{S}\right\}}\right] d t .
$$

Proof. See Appendix I.

Note that the last term on the right-hand side (RHS) of (8) indicates that the incremental gain over the time interval $[t, t+d t]$ from exercising the option at $t$ is $\left(r K-\delta S_{t}\right) d t$. Similarly, the incremental gain from exercising a call option whose payoff is $\left(S_{t}-K\right)^{+}$is $\left(\delta S_{t}-r K\right) d t$. Since this gain becomes negative when $\delta=0$, an American barrier call option should not be exercised before expiration unless there is some kind of compensation for the absence of the dividend. ${ }^{7}$

Eq. (8) provides an analytical representation for the price of an American "up-and-out" put option. However, in order to facilitate the implementation of the formula, it would be desirable to have an explicit expression for the expectation $E[\cdot]$ in (8). This, in turn, depends on the shape of the optimal exercise boundary $\partial \mathcal{C}$. We assume that the boundary can be represented by a continuous function $B_{\mathrm{uo}}^{p}:[0, T] \rightarrow \mathcal{R}_{++}{ }^{8}$

Corollary 1 Suppose the underlying asset pays no dividend. ${ }^{9}$ The price of an American "up-and-out" put option with the barrier level $H>K$ is given by

$$
P_{\mathrm{uo}}\left(S_{0}, K\right)=p_{\mathrm{uo}}\left(S_{0}, K\right)+\int_{0}^{T} r K e^{-r t} \operatorname{Pr}\left(S_{t} \leq B_{\mathrm{uo}, t}^{p} ; M_{0}^{t}<H\right) d t
$$

where $\operatorname{Pr}(\cdot)$ is the risk-neutral probability, $M_{t_{1}}^{t_{2}}$ is the running maximum as defined before, and the argument $\left(S_{0}, K\right)$ is used to emphasize that the option is valued at time 0 with the underlying asset price equal to $S_{0}$ and a strike price $K$.

The optimal exercise boundary $\mathcal{B}_{\mathrm{uo}}^{p}=\left\{B_{\mathrm{uo}, t}^{p} ; t \in[0, T]\right\}$ is determined by the following condition

$$
K-B_{\mathrm{uo}, t}^{p}=\lim _{S_{t} \downarrow B_{\mathrm{uo}, t}^{p}} P_{\mathrm{uo}}\left(S_{t}, K\right) ; \quad M_{0}^{t}<H, \quad \forall t \in[0, T]
$$

Proof. We have that the exercise event $\left\{\left(S_{t}, t\right) \in \mathcal{S}_{t}\right\}=\left\{S_{t} \leq B_{\mathrm{uo}, t}^{p}\right\}$ and dividend $\delta=0$. Substituting these into (8) yields (9).

We now provide further analytical results for the case of out-of-the-money "up-and-out" put options, on which the implementation (discussed later on in section 3) is based.

\footnotetext{
${ }^{7}$ Merton (1973) first pointed this out in the case of the American options, both standard and "downand-out."

${ }^{8}$ This amounts to assuming that the boundary consists only of a single piece. This is expected to hold given that the underlying follows a geometric Brownian motion. Our numerical studies also support the validity of the assumption (c.f. Tables 1 and 2 and Figure 1). However, a rigorous justification of this assumption remains to be provided.

${ }^{9}$ The case of a non-zero dividend yield is considered in the proof of the general formula in Appendix I.
} 
We define the notation as follows:

$$
\begin{aligned}
\mu & \equiv r-\sigma^{2} / 2 \\
\lambda & \equiv \frac{r+\sigma^{2} / 2}{\sigma^{2}} \\
d_{2}(x, y, t) & \equiv \frac{\ln (x / y)+\mu t}{\sigma \sqrt{t}} \\
d_{1}(x, y, t) & \equiv d_{2}(x, y, t)+\sigma \sqrt{t}
\end{aligned}
$$

where $\sigma$ denotes the volatility of the instantaneous return in the underlying asset.

\subsubsection{Option Prices and Hedge Parameters}

As discussed before, the price of an American "up-and-out" put option can be written as follows:

$$
P_{\mathrm{uo}}\left(S_{0}, K\right)=p_{\mathrm{uo}}\left(S_{0}, K\right)+P_{\mathrm{uo}}^{p}\left(S_{0}, K\right)
$$

where $p_{\mathrm{uo}}$ and $P_{\mathrm{uo}}^{p}$ are the prices of the corresponding European option and the early exercise premium respectively. Specifically, the price of the European "up-and-out" put option can be written as [see Rubinstein and Reiner (1991)]:

$$
\begin{aligned}
p_{\text {uo }}\left(S_{0}, K\right) & =p^{o}\left(S_{0}, K\right)-p_{\text {ui }}\left(S_{0}, K\right) \\
& =p^{o}\left(S_{0}, K\right)-\left(H / S_{0}\right)^{2 \lambda-2} p^{o}\left(H^{2} / S_{0}, K\right)
\end{aligned}
$$

where $p^{o}(x, K)$ denotes the Black-Scholes price of a standard European put option with current underlying price $x$ and strike price $K$, and $p_{\text {ui }}(\cdot)$ represents the price of a European up-and-in put option. Following Black and Cox (1976) (Eq. [7]) (see also Cox and Miller (1965, p.221, Eq. [71])], we have

$$
\operatorname{Pr}\left(S_{t} \leq B_{\mathrm{uo}, t}^{p} ; M_{0}^{t}<H\right)=N\left(-d_{2}\left(S_{0}, B_{\mathrm{uo}, t}^{p}, t\right)\right)-\left(H / S_{0}\right)^{2 \lambda-2} N\left(-d_{2}\left(H^{2} / S_{0}, B_{\mathrm{uo}, t}^{p}, t\right)\right)
$$

where $N(\cdot)$ represents the cumulative standard normal function. Thus the American premium of an "up-and-out" put option is given by

$$
P_{\mathrm{uo}}^{p}=\int_{0}^{T} e^{-r t} r K\left[N\left(-d_{2}\left(S_{0}, B_{\mathrm{uo}, t}^{p}, t\right)\right)-\left(H / S_{0}\right)^{2 \lambda-2} N\left(-d_{2}\left(H^{2} / S_{0}, B_{\mathrm{uo}, t}^{p}, t\right)\right)\right] d t
$$

Notice that the first term on the RHS of (13) is the exercise premium of a standard American put option and, as expected, the second term on the RHS goes to zero as $H \uparrow \infty$.

The hedge parameters can be calculated in a straightforward fashion from (11). For instance, the delta is

$$
\Delta_{\mathrm{uo}}^{p}=\frac{\partial}{\partial S_{0}}\left(p_{\mathrm{uo}}+P_{\mathrm{uo}}^{p}\right)
$$


where (for the European part)

$$
\begin{aligned}
\frac{\partial p_{\mathrm{uo}}}{\partial S_{0}}= & -N\left(-d_{1}\left(S_{0}, K, T\right)\right)-\left(H / S_{0}\right)^{2 \lambda}\left[N\left(-d_{1}\left(H^{2} / S_{0}, K, T\right)\right)\right. \\
& \left.-(2 \lambda-2) p^{o}\left(H^{2} / S_{0}, K\right) /\left(H^{2} / S_{0}\right)\right]
\end{aligned}
$$

and (for the American premium part)

$$
\begin{aligned}
\frac{\partial P_{\mathrm{uo}}^{p}}{\partial S_{0}}= & -\int_{0}^{T} e^{-r t} \frac{r K}{S_{0} \sigma \sqrt{t}}\left\{n\left(d_{2}\left(S_{0}, B_{\mathrm{uo}, t}^{p}, t\right)\right)+\left(H / S_{0}\right)^{2 \lambda-2}\right. \\
& {\left.\left[n\left(d_{2}\left(S_{0}, H^{2} / B_{\mathrm{uo}, t}^{p}, t\right)\right)-(2 \lambda-2) \sigma \sqrt{t} N\left(-d_{2}\left(H^{2} / S_{0}, B_{\mathrm{uo}, t}^{p}, t\right)\right)\right]\right\} d t }
\end{aligned}
$$

In the above, $n(\cdot)$ is the standard normal density function. One can show that, similar to the option price, the delta of an American "up-and-out" option also collapses to that of a standard American option as the barrier goes to infinity. Formulae for other hedge parameters (e.g. gamma, vega, rho, etc.) can be obtained similarly by differentiating (11) accordingly and are not presented here in the interest of brevity.

It has been generally recognized that the hedging of barrier options is more difficult than that of standard options. ${ }^{10}$ This is mainly due to the unstable properties of the hedge parameters of barrier options, especially near the barrier. The formulae developed here allow us to analytically examine these properties and provide an approach to computing the hedge parameters that is free of the "near-barrier" problem.

\subsubsection{The Optimal Exercise Boundary}

We now examine the properties of the optimal exercise boundary. It follows from (10) that the boundary $\left\{B_{\mathrm{uo}, t}^{p} ; t \in[0, T]\right\}$ is determined by the following condition

$$
K-B_{\mathrm{uo}, t}^{p}=P_{\mathrm{uo}}\left(B_{\mathrm{uo}, t}^{p}, K\right) ; \quad B_{\mathrm{uo}, t}^{p}<H, \quad \forall t \in[0, T) .
$$

Using (11) and (12) yields

$$
\begin{aligned}
& K-B_{\mathrm{uo}, t}^{p}=p_{\mathrm{uo}}\left(B_{\mathrm{uo}, t}^{p}, K\right)+\int_{t}^{T} d s r K e^{-r(s-t)} \\
& \quad\left[N\left(-d_{2}\left(B_{\mathrm{uo}, t}^{p}, B_{\mathrm{uo}, s}^{p}, s-t\right)\right)-\left(\frac{H}{B_{\mathrm{uo}, t}^{p}}\right)^{2 \lambda-2} N\left(-d_{2}\left(H^{2} / B_{\mathrm{uo}, t}^{p}, B_{\mathrm{uo}, s}^{p}, s-t\right)\right)\right]
\end{aligned}
$$

In the context of standard American options with a log-normal price process for the underlying asset, van Moerbeke (1976) proves that the exercise boundary is continuously differentiable, and Jacka (1991) and Kim (1990) discuss its monotonicity in time. We now demonstrate that the exercise boundary (for both standard and non-standard options) has

\footnotetext{
${ }^{10}$ Derman, Ergener, and Kani (1995) and Carr, Ellis, and Gupta (1998) demonstrate that one can use the property of put-call parity to construct a portfolio consisting of a put and a call to statically hedge European barrier options.
} 
two additional properties. One is homogeneity of degree one in the strike price and the barrier level and the other is translational invariance in time. As shown later, these two properties, combined with the fact that the boundary is independent of the underlying asset price, have important implications for the implementation of a pricing model for American options.

Theorem 2 For American barrier options with a strike level $K$ and a barrier $H$, the optimal exercise boundary has the following properties:

(a) (Homogeneity of Degree One in Strike and Barrier Prices)

$$
B_{\mathrm{uo}, t}^{\mathrm{p}}(\lambda K, \lambda H)=\lambda B_{\mathrm{uo}, t}^{\mathrm{p}}(K, H) \quad \forall \lambda>0, t \in[0, T]
$$

(b) (Translational Invariance)

$$
B_{\mathrm{uo}, t-\left(T_{2}-T_{1}\right)}^{\mathrm{p}}\left(K, H, T_{1}\right)=B_{\mathrm{uo}, t}^{\mathrm{p}}\left(K, H, T_{2}\right) \quad \forall t \in\left[T_{2}-T_{1}, T_{2}\right]
$$

(c) (Monotonicity in Time)

$$
\partial B_{\mathrm{uo}, t}^{\mathrm{p}} / \partial t>0, t \in[0, T)
$$

(d) (Monotonicity in the Barrier Level)

$$
\partial B_{\mathrm{uo}, t}^{\mathrm{p}} / \partial H<0, t \in[0, T]
$$

Proof. See Appendix II.

From the proofs, one can see that the translational invariance in time should hold for any American option with a stationary process for the underlying asset price. The monotonicity is valid as long as the reward for stopping equals $K-S_{t}$. The homogeneity follows from the homogeneity of the option pricing function and relies on the log-normality assumption on the underlying process and the assumption that the payoff function $h(\cdot)$ is homogeneous of degree one in $(K, H)$.

For the sake of completeness, we state the following corollary without proof.

Corollary 2 For standard American (put) options with a strike level $K$, the optimal exercise boundary has the following properties:

(a) (Homogeneity in Strike)

$$
B_{t}^{\mathrm{p}}(\lambda K)=\lambda B_{t}^{\mathrm{p}}(K) \quad \forall \lambda>0, t \in[0, T]
$$

(b) (Translational Invariance)

$$
B_{t-\left(T_{2}-T_{1}\right)}^{\mathrm{p}}\left(K, T_{1}\right)=B_{t}^{\mathrm{p}}\left(K, T_{2}\right) \quad \forall t \in\left[T_{2}-T_{1}, T_{2}\right]
$$

(c) (Monotonicity in Time)

$$
\partial B_{t}^{\mathrm{p}} / \partial t>0, t \in[0, T)
$$


Theorem 2 and Corollary 2 show the sufficiency of the log-normality assumptions for the homogeneity of the optimal exercise boundary (and the homogeneity of the option pricing function). Whereas the sufficiency has been discussed in the literature (see below), to the best of our knowledge, necessity has not been established. Indeed, the homogeneity of the pricing function is sometimes assumed to hold in order to simplify the problem. ${ }^{11}$

\subsubsection{Put-Call "Symmetry"}

Chesney and Gibson (1995) and McDonald and Schroder (1998) show that a put-call "symmetry" condition holds for standard American options. ${ }^{12}$ Namely,

$$
\begin{aligned}
C\left(S_{t}, K, \delta, r\right) & =P\left(K, S_{t}, r, \delta\right) \\
B_{t}^{\mathrm{c}}(K, r, \delta) & =\frac{K^{2}}{B_{t}^{\mathrm{p}}(K, \delta, r)},
\end{aligned}
$$

where $B_{t}^{\mathrm{c}}(\cdot)$ and $B_{t}^{\mathrm{p}}(\cdot)$ denote the optimal exercise boundary point at time $t$ of a standard American call and put option, respectively. Notice that, given $K, \delta, r, \sigma$ and $t$, both $B_{t}^{\mathrm{c}}(\cdot)$ and $B_{t}^{\mathrm{p}}(\cdot)$ are independent of the spot price $S_{t}$. In other words, the exercise decision is made independently of the current spot price. We now demonstrate that a similar relation holds for American barrier options.

Theorem 3 Under Assumption 2, for barrier options there exists a put-call "symmetry" between a "down-and-out" call option and an "up-and-out" put option, i.e., the following relationships hold

$$
\begin{aligned}
C_{\mathrm{do}}\left(S_{0}, K, H, r, \delta\right) & =P_{\mathrm{uo}}\left(K, S_{0}, K S_{0} / H, \delta, r\right) ; \\
B_{\mathrm{do}, t}^{\mathrm{c}}(K, H, r, \delta) & =\frac{K^{2}}{B_{\mathrm{uo}, t}^{\mathrm{p}}\left(K, K^{2} / H, \delta, r\right)}
\end{aligned}
$$

where the superscripts $\mathrm{c}$ and $\mathrm{p}$ denote call and put, respectively.

Proof. See Appendix III.

The intuition behind this "symmetry" relation is as follows. We know that the put-call "symmetry" holds for standard options. For "knock-out" options, the additional feature is

\footnotetext{
${ }^{11}$ To some extent, the implication of the homogeneity of the optimal exercise boundary on the underlying price process can be studied by examining the possible restrictions on the underlying process imposed by the homogeneity of the option pricing function. This is because the former homogeneity comes from the latter homogeneity. Furthermore, to study the necessary conditions for homogeneity, it is enough to consider the case of European options.

As shown in Merton (1973), for a standard European option, a return distribution that is independent of the initial price level is, in general, sufficient for the option price to be homogeneous of degree one in $(S, K)$. [Merton (1990, p.306-307) provides a counter example for this sufficiency condition.] We conjecture that this condition on the return distribution may also be necessary for the homogeneity of the option price in a one-factor continuous-time setting.

${ }^{12}$ See also Schroder (1997).
} 
the "knock-out" provision. Hence, the difference between the value of a "knock-out" option and the value of the corresponding standard option depends only on the likelihood of the asset price breaching the barrier. The likelihood of breaching the barrier is determined by the distance between the stock price and the barrier, and the drift of stock price. Under the assumption that the stock price follows a log-normal diffusion, the asset price of the "down-and-out" call drifts away from the barrier at the speed of $r-\delta$. For the put option, the drift is $\delta-r$. Since the barrier is above the stock price in this case, the stock price again drifts towards the barrier at the speed of $\delta-r$, in another words, away from the barrier at the speed of $r-\delta$, the same speed as in the call option case. Given that the drifts in the two cases are the same, we also require that the distances between the logarithm of the stock price and the logarithm of the barrier be the same. For the call option, the distance is $\ln S-\ln H$, and for the put option, the distance is $\ln H^{\mathrm{p}}-\ln K$, where $H^{\mathrm{p}}$ is the equivalent barrier for the put option. Equating the two yields $H^{\mathrm{p}}=S K / H$. Similar equivalent arguments also apply to the optimal exercise condition.

Note that, in principle, log-normality is a sufficient, but not a necessary condition for put-call "symmetry" to hold. However, the "symmetry" requires that a strong restriction be placed on the underlying distribution even in the zero-drift case. In fact, as shown in Carr, Ellis and Gupta (1998), the diffusion term has to have a "symmetry" around the current asset price for the argument to go through.

\subsection{In-the-Money "Up-and-Out" Puts}

In this subsection, we consider in-the-money (at-the-money) American "up-and-out" put options. Consider first the case of zero dividend yield. Here, we have:

Theorem 4 If the dividend yield on the underlying asset is zero, that is $\delta=0$, an in-themoney (at-the-money) "up-and-out" American put option will always be exercised either before it expires or at expiration.

Proof. See Appendix IV.

As in the case of any put option, early exercise allows the holder of the option to capture the time value of money on the early receipt of the exercise price, by giving up the insurance value of the option and the present value of the dividend stream on the underlying asset. As long as there is no insurance value or stream of dividends, this type of put option should be exercised if it is in the money. This is exactly what happens in this case.

It is worth mentioning that Theorem 4 does not carry over to the case of out-of-themoney "knock-out" options. This is because when $H>K$, an exercised position may not have enough cash to cover the short position in the stock when the barrier is breached. ${ }^{13}$

\footnotetext{
${ }^{13}$ See case (a) of Appendix IV.
} 
This observation also shows that the option will be exercised unconditionally, if the rebate amount, $R$, is less than $K-H$, when $K>H$.

Now suppose $\delta>0$. In this case, it may not be always optimal to exercise an inthe-money "up-and-out" put since the incremental gain over some time-interval $d t$ from exercising the option may be negative. However, we expect Theorem 4 to also hold in the case of "low" dividend yield because of continuity and, in particular, in the case of $\delta \ll r$.

\subsection{Other Types of Barrier Options}

So far, in this section, we have examined "up-and-out" put options and "down-and-out" call options. We now briefly analyze "up-and-out" call and "down-and-out" put options. As shown below, these options include "capped" options as special cases. ${ }^{14}$

Consider the case of American "up-and-out" call options. At-the-money $(H=K)$ and out-of-the-money $(H<K)$ calls are easy to analyze. One can see that the only possible cashflows from these options come from rebate at the barrier. As a result, the option value is equal to the discounted rebate times the risk-neutral probability of the underlying price hitting the barrier.

The analysis of in-the-money "up-and-out" calls $(H>K)$ is more involved. Suppose the dividend yield $\delta$ is zero. Like a standard American call option, an American "up-andout" call on a non-dividend-paying stock should not be exercised early. This can also be seen from the discussion of Theorem 1. As a result, one should exercise an American "upand-out" call option at time $t$ only when $S_{t}=H$. Namely, the optimal exercise boundary coincides with the barrier. Denote the optimal exercise boundary of American "up-andout" call options by $\mathcal{B}_{\mathrm{uo}}^{\mathrm{c}} \equiv\left(B_{\mathrm{uo}, t}^{\mathrm{c}}\right)_{t \in[0, T]}$. We have $B_{\mathrm{uo}, t}^{\mathrm{c}}=H \forall t \in[0, T]$. The payoff at the barrier equals $H-K$. This exercise strategy is optimal as long as the rebate $R \leq H-K$. However, if $R>H-K$, then one should never exercise early. In either case, however, the option value is equal to the value of a European barrier option with an effective rebate $R^{\prime}=\max (R, H-K)$.

Now suppose $\delta>0$. In this case, the option payoff upon exercise equals $(\min (S, H)-$ $K)^{+}=\min \left[(S-K)^{+}, H-K\right]$. This payoff is the same as that of an American "capped" call with a constant cap $H$. Let $\mathcal{B}^{\mathrm{c}} \equiv\left(B_{t}^{\mathrm{c}}\right)_{t \in[0, T]}$ be the exercise boundary of an otherwise identical standard American call option. We have

Theorem 5 Consider an American "up-and-out" call option with $H>K{ }^{15}$ If the rebate at the barrier is no more than $H-K$, then such a call option is equivalent to an American

\footnotetext{
${ }^{14}$ See, for example, Boyle and Turnbull (1989) and Broadie and Detemple (1995) on "capped" call options.

${ }^{15}$ Technically, for the knock-out event and the exercise date to be well defined, the option contract is defined in a way such that when the asset price first touches the barrier, the option holder has the option to either exercise or let the option be knocked out.
} 
"capped" call option with a cap equal to $H$. Furthermore, the exercise boundary of the "up-and-out" call option is

$$
B_{\mathrm{uo}, t}^{\mathrm{c}}=\min \left(H, B_{t}^{\mathrm{c}}\right) \quad \forall t \in[0, T]
$$

and the option value is given by

$$
C_{\mathrm{uo}}(t)=c_{\mathrm{uo}}(t)+E_{t}\left[\int_{t}^{\tau_{H}} e^{-r(u-t)}\left(\delta S_{u}-r K\right) I_{\left\{S_{u} \geq B_{\mathrm{uo}, u}^{\mathrm{c}}\right\}} d u\right], \quad t \in\left[0, \tau_{H}\right]
$$

where $c_{\mathrm{uo}}(t)$ denotes the value of the corresponding European "up-and-out" call option with rebate $H-K$, and the "hitting time" $\tau_{H}$ is defined as follows

$$
\tau_{H}=\inf \left\{t \in[0, T] \mid S_{t} \geq H\right\}
$$

$\tau_{H}=T$ if the event does not occur by $T$.

Proof. Given that rebate $R \leq H-K$, it is always better to exercise an "up-and-out" call than to wait for a knock-out. This strategy guarantees a payoff of $H-K$ at $H$. It is then obvious that "up-and-out" call and "capped" call options are equivalent. Eq. (30) follows from Theorem 1 in Broadie and Detemple (1995). The representation in (31) can be obtained via a reparametrization of the pricing formula (4.4) in Broadie and Detemple (1997) for American "capped" exchange options with proportional cap. ${ }^{16}$

Notice that both (30) and (31) apply also to the case of zero dividend. The explicit formula for the European price $c_{\mathrm{uo}}(t)$ can be found in Rubinstein and Reiner (1991). Since $\tau_{H}$ is, in general, random, the expectation in (31) might not be always carried out explicitly.

The intuition behind Theorem 5 can be conveyed by considering three cases for the location of the barrier level $H$ relative to the level of the boundary $\mathcal{B}^{\mathrm{c}}$.

- Case (a): $H>B_{t}^{\mathrm{c}} \forall t \in[0, T]$. First, note that when $H \uparrow \infty$, an "up-and-out" call option is equivalent to an otherwise identical standard American call option, since the probability of being knocked-out goes to zero. As a result, the two options have the same exercise boundary and hence the same value, i.e., $\lim _{H \uparrow \infty} \mathcal{B}_{\mathrm{uo}}^{\mathrm{c}}=\mathcal{B}^{\mathrm{c}}$ and $\lim _{H \uparrow \infty} C_{\mathrm{uo}}(S, K, H)=C(S, K)$, where $\mathcal{B}^{\mathrm{c}} \equiv\left(B_{t}^{\mathrm{c}}\right)_{t \in[0, T]}$ denotes the exercise boundary of a standard American call option.

- Case (b): $H \in\left[B_{T}^{\mathrm{c}}, B_{0}^{\mathrm{c}}\right]$. Note that $B_{t}^{\mathrm{c}}$ is a decreasing function of $t$, due to the declining insurance value and time value of money of the exercise price, reaching a minimum at the expiration date $T$, where $B_{T}^{\mathrm{c}}=\max (r K / \delta, K) \cdot{ }^{17}$ Assuming that the boundary is continuous, it intersects the barrier level $H$ at a unique time $t^{*} \in[0, T]$,

\footnotetext{
${ }^{16}$ We thank a referee for pointing this out.

${ }^{17}$ See Kim (1990) and Jacka (1991) for details.
} 
s.t. $B_{t^{*}}^{\mathrm{c}}=H$. It follows that an "up-and-out" call alive at time $t \in\left[t^{*}, T\right]$ is equivalent to a standard American call option by the argument for Case (a) above. This implies that $B_{\mathrm{uo}, t}^{\mathrm{c}}=B_{t}^{\mathrm{c}} \forall t \in\left[t^{*}, T\right]$. In particular, $B_{\mathrm{uo}, t^{*}}^{\mathrm{c}}=H$. Since $B_{\mathrm{uo}, t}^{\mathrm{c}}$ is a decreasing function of $t, B_{\mathrm{uo}, t}^{\mathrm{c}} \geq H \forall t \in\left[0, t^{*}\right]$. However, unless exercised, an "up-and-out" call will be knocked out at $H$. This indicates that $B_{\mathrm{uo}, t}^{\mathrm{c}}=H \forall t \in\left[0, t^{*}\right]$. It follows that $B_{\mathrm{uo}, t}^{\mathrm{c}}=\min \left[H, B_{T}^{\mathrm{c}}\right] \forall t \in[0, T]$.

- Case (c): $H<B_{T}^{\mathrm{c}}$. Recall that $B_{\mathrm{uo}, T}^{\mathrm{c}}=\min [H, \max (r K / \delta, K)]$. Since $B_{T}^{\mathrm{c}}=$ $\max (r K / \delta, K)$, it follows that $B_{\mathrm{uo}, T}^{\mathrm{c}}=H$. Therefore, $\mathcal{B}^{\mathrm{c}}$ lies above the barrier everywhere since $B_{t}^{\mathrm{c}}$ declines with $t$ and reaches a minimum at $T$.

Since $B_{\mathrm{uo}, t}^{\mathrm{c}}$ is a decreasing function of $t, B_{\mathrm{uo}, t}^{\mathrm{c}}=H \forall t \in[0, T]$. In this case, an American "up-and-out" call behaves like a European "up-and-out" call, but with a rebate $R=H-K$. Thus, the case is similar to the case where $\delta=0$.

American "down-and-out" put options can be analyzed in a similar fashion. Consider first out-of-the-money $(H>K)$ and at-the-money $(H=K)$ put options. Like out-of-themoney and at-the-money "up-and-out" calls, cashflows from these options come only from rebate at the barrier. The valuation problem, is therefore, straightforward. Next, consider in-the-money $(H<K)$ "down-and-out" put options. As expected, these options with a rebate $R \leq K-H$ are equivalent to American "capped" puts with a cap equal to $H$. Let $\mathcal{B}^{\mathrm{p}} \equiv\left(B_{t}^{\mathrm{p}}\right)_{t \in[0, T]}$ and $\mathcal{B}_{\mathrm{do}}^{\mathrm{p}} \equiv\left(B_{\mathrm{do}, t}^{\mathrm{p}}\right)_{t \in[0, T]}$ denote respectively the exercise boundary of standard American put and American "down-and-out" put options. We can now state and prove:

Theorem 6 Consider an American "down-and-out" put option with strike $K$, expiration date $T$, and barrier level $H<K$. If the rebate at $H$ is no more than $K-H$, then the option can be considered to be an American "capped" put option with a cap equal to $H$. Furthermore, the optimal exercise boundary of the "down-and-out" put option can be characterized as follows

$$
B_{\mathrm{do}, t}^{\mathrm{p}}=\max \left(H, B_{t}^{\mathrm{p}}\right) \quad \forall t \in[0, T]
$$

Proof. This theorem follows from arguments similar to those in the above discussion of Theorem 5.

When $H<B_{t}^{\mathrm{p}}$ forallt $\in[0, T]$, the put will be exercised before the asset price touches the barrier. Hence, $B_{\mathrm{do}, t}^{\mathrm{p}}=\max \left(H, B_{t}^{\mathrm{p}}\right)=B_{t}^{\mathrm{p}}$.

When $H>B_{T}^{\mathrm{p}}=r K / \delta$, where $r<\delta$, it is easy to see that $B_{\mathrm{do}, T}^{\mathrm{p}}=H$. Since $B_{\mathrm{do}, t}^{\mathrm{p}}$ is a non-decreasing function in $t, B_{\mathrm{do}, t}^{\mathrm{p}}=H \forall t \in[0, T]$. In this case, the option becomes a European "down-and-out" put with rebate $K-H$.

When $H \in\left[B_{T}^{\mathrm{p}}, B_{0}^{\mathrm{p}}\right]$, the barrier intersects $\mathcal{B}^{\mathrm{p}}$. Recall that $B_{t}^{\mathrm{p}}$ increases in $t$. It follows that there exists a unique $t_{\mathrm{do}}^{*}=\left\{t \in(0, T) \mid B_{t}^{\mathrm{p}}=H\right\}$. One can see in the time interval 
$\left[t_{\mathrm{do}}^{*}, T\right]$ a live American "down-and-out" put option is, in fact, equivalent to a standard American put option. Since $B_{t_{\mathrm{do}}^{*}}^{\mathrm{p}}=H$ and $B_{\mathrm{do}, t}^{\mathrm{p}}$ is monotonic in $t, B_{\mathrm{do}, t}^{\mathrm{p}}=H \forall t \in\left[0, t_{\mathrm{do}}^{*}\right]$.

The valuation problem in this case can be handled similarly to the case of "up-and-out" put options in section 2.1. More specifically, a formula similar to (31) can be obtained for pricing American "down-and-out" put options. A symmetry relation between American "up-and-out" call and "down-and-out" put options similar to (28) and (29) can be also established. Detailed discussions, however, are omitted here for the sake of brevity.

\section{Implementation and Numerical Results}

In this section, we focus on American "up-and-out" put options written on non-dividend paying assets and discuss the implementation of the pricing and hedging formulae (11) and (14) given in section 2.1.1. We then report some numerical results to illustrate the efficiency and accuracy of our implementation scheme.

\subsection{Implementation}

The implementation involves two steps. The first is to compute the optimal exercise boundary $\mathcal{B}$. The second is to calculate the option prices or hedge ratios taking $\mathcal{B}$ as input.

Since $\mathcal{B}$ is implicitly defined by the integral equation (18), the boundary has to be calculated numerically. Various numerical schemes have been proposed for this purpose in the context of standard American options on non-dividend paying assets. One such scheme is to compute the boundary recursively, an idea originally suggested by Kim (1990). Starting

with $B_{T}, B_{T-1}$ is calculated from (18). Next, $B_{T-2}$ is calculated, also from (18), taking $B_{T}$ and $B_{T-1}$ as inputs. This procedure is repeated iteratively until the entire exercise boundary (an approximated one, strictly speaking) is generated. Once the optimal exercise boundary is obtained, the calculation of option prices and hedge ratios is straightforward, involving only a univariate numerical integration. However, this recursive scheme, which is somewhat computation-intensive, can be accelerated using analytical approximations of the exercise boundary, at least for the purpose of pricing.

We focus on two approximation schemes developed in the context of standard American options and based on the integral representation of the early exercise premium. One method is to approximate the exercise boundary by a step-function, i.e. to replace the integral in (18) by a simple sum in our case. This is the approach taken in Huang, Subrahmanyam, and $\mathrm{Yu}$ (1996). The other method is to approximate the exercise boundary by an exponential function. This is based on the observation that the exercise boundary of standard American put options has a shape similar to that of an exponential function. Omberg (1987) and Ho, Stapleton, and Subrahmanyam (1994; 1997b) use a single-piece 
exponential function to approximate the exercise boundary. Ju (1998) uses a multi-piece exponential (MPE) function approximation and also utilizes the integral representation of the early exercise premium. ${ }^{18}$ Under both schemes, the approximated boundary can be described by a few parameters. This allows us to directly compute only a few points on the exercise boundary and, as a result, can increase considerably the computational efficiency. The resulting option prices/hedge ratios can then be used to extrapolate the true price/hedge ratios to improve the accuracy of the two schemes (c.f. Appendix V for more details).

\subsubsection{A "Tabulation" Approach to Pricing Options}

The implementation procedure described previously also allows for a scheme to increase computational efficiency in the valuation of multiple contracts written on the same underlying asset.

On a given day, traders typically need to evaluate their options positions several times. This involves computing positions of contracts written on the same underlying asset. These contracts differ only in their strike price, barrier level and time to expiration. A conventional implementation scheme involves the calculation of the exercise boundary for each contract, i.e., for each value of the parameter set $\left(S_{t}, K, T-t, \sigma, r, H\right)$. However, due to its homogeneity and translational invariance properties, the exercise boundary needs to be calculated for only a few values of the parameter set. This avoids some of the problems of repetitive computation of option prices and hedge ratios. As a result, the computational time can be reduced significantly when pricing a basket of options written on the same underlying asset. ${ }^{19}$ The translational invariance property implies that, among all the contracts considered [characterized by the parameter set $\left.\left(S_{t}, K, T-t, \sigma, r, H\right)\right]$, only the boundary for the longest $T-t$, ceteris paribus, needs to be calculated. The homogeneity property suggests that among all the contracts considered, ceteris paribus, for standard American options only the boundary for one value of $K$, needs to be calculated, and for American barrier options among the contracts with the same proportional value of $(K, H)$, only the boundary for one set of $(K, H)$ needs to be calculated. ${ }^{20}$

These observations suggest an approach to the valuation and hedging of American options in which the optimal exercise boundary is tabulated for different values of the pa-

\footnotetext{
${ }^{18}$ Other recent work on standard American options includes, but not limited to, Breen (1991), Broadie and Detemple (1996), Bunch and Johnson (1992), Carr (1998), Carr and Faguet (1995).

${ }^{19}$ Similar ideas are independently developed in Joubert and Rogers (1997), whose work we were not aware of until several early drafts of our paper were completed.

${ }^{20}$ Another advantage of computing the exercise boundary first is that, given a contract, one can easily determine if it is optimal to exercise right away at the valuation time, say $t_{0}$. Given the exercise boundary point at $t_{0}, B_{t_{0}}$, one would exercise the option if $S_{t_{0}} \leq B_{t_{0}}$. In contrast, the use of alternative methods would require the computation of the option value at $t_{0}$ to make the decision. See also Brealey, Hodges, and Selby (1982) and Selby (1983) for a related discussion.
} 
rameter set $(K, H, T-t, \sigma, r)$. Computing the option prices and hedge ratios then amounts to calling a tabulated "exercise boundary" function.

\subsubsection{Optimal Exercise Boundary}

As mentioned earlier, the implementation of the analytic method requires the optimal exercise boundary as an input. In this section, as an illustration, we provide plots of the optimal exercise boundary for American barrier put options. The boundary for American barrier call options can be obtained using the put-call "symmetry" relationship derived earlier. As shown below, useful information can be extracted from such a plot of the optimal exercise boundary.

Figure 1 illustrates the plots of the optimal exercise boundary for American "up-andout" put options on non-dividend-paying stocks for different levels of the barrier. Specifically, we choose six levels of the barrier, namely, $H=45,45.01,45.10,46,50,100$. The values of the other relevant parameters are $K=45, T-t=1, \sigma=0.2$, and $r=0.0488$. One can see that for a given $H$, the exercise boundary divides the domain into two regions. The region above the boundary is called the continuation region, $\mathcal{C}$, in which exercise is not optimal, and the region below is the stopping region, $\mathcal{S}$, where it pays to exercise early. The boundary with $H=100$ is essentially the same as the boundary of an otherwise identical standard American put option $(H=\infty)$. One can see from the figure that as $H$ decreases, ceteris paribus, the optimal exercise boundary moves upward, or equivalently the size of the stopping region increases. This indicates that the American feature of an "up-and-out" put option becomes more valuable as $H$ gets higher. One interesting result obtained from plotting the optimal exercise boundary is that in the case where the dividend yield is zero, it is always optimal to exercise early an American "up-and-out" put option, when the barrier level is equal to the strike price. This is a direct result of Theorem 4. One can see from Figure 1 that the optimal exercise boundary with $H=45=K$ coincides with the line, $K=45$. This implies that the "up-and-out" option should always be exercised because the setup dictates that the underlying asset price is below the strike price.

Figure 2 illustrates the price homogeneity of the optimal exercise boundary for American out-of-the-money "up-and-out" put options on non-dividend-paying stocks. Figure 2(a) shows plots of the boundary with $(K=45, H=50)$, the solid curve, and the boundary with ( $K=90, H=100)$, the dashed curve, to illustrate the homogeneity in $(K, H)$. Figure 2(b) shows plots of the boundary with $(K=45, H=100)$, the solid curve, and the boundary with $(K=90, H=500)$, the dashed curve, to illustrate the homogeneity in $K$ when $H \gg K$. Note that when $H \gg K$, an "up-and-out" put option is essentially equivalent to a standard American put option. So Figure 2(b) actually illustrates the homogeneity in $K$ of optimal exercise boundaries for standard American options. The values of other relevant parameters are time to expiration, $T-t=1$ (year), volatility, $\sigma=0.2$, and risk-free rate, $r=0.0488$. In both (a) and (b), the height of the dashed curve is twice the height of the 
solid curve, which verifies the price homogeneity.

Figure 3 illustrates the translational invariance of the optimal exercise boundary of American out-of-the-money "up-and-out" put options on non-dividend-paying stocks. Two plots of the boundary are shown in the figure and differ only in time to expiration, the dashed curve with $T-t=0.5$ (year) and the solid curve with $T-t=1$ (year). The values of other relevant parameters are strike $K=45$, barrier $H=50$, volatility $\sigma=0.2$, and risk-free rate $r=0.0488$. When shifted to the left for $T-t=0.5$, the solid curve will coincide with the dashed curve, which verifies the stationarity property.

We should emphasize that the method developed here has a definite advantage over the lattice methods in computing the optimal exercise boundary. For instance, it would be very difficult to obtain a plot as smooth as those shown in Figure 1 using a lattice method, even with a large number of time steps. In contrast, the plots shown in Figure 1, for instance, were generated using the analytic formula with 200 points (time-steps) and the amount of the computational time required is about 0.6 seconds (CPU time) on a Sun Ultra 1 workstation.

\section{$3.2 \quad$ Numerical Results}

It has been recognized that the simple binomial method is not appropriate for pricing barrier options due to the fact that the price of such options is very sensitive to the location of the barrier in the lattice. The reason for this sensitivity comes from the fact that the option-value function is not smooth around the barrier. The existence of such "kinks" and the discrete price-space in the binomial/trinomial models effectively causes a shift of the barrier to a nearby layer of nodes, once the barrier falls in-between two layer of nodes. ${ }^{21}$

In this section, we illustrate the accuracy and efficiency of the pricing formula (11) in relation to some existing lattice methods which modify the standard binomial method. The three such methods that we are aware of for pricing barrier options, are the restricted binomial/trinomial methods of Boyle and Lau (1994), and Ritchken (1995), the trinomial method of Cheuk and Vorst (1996), and the adaptive mesh method of Gao (1996). Boyle and Lau, and Ritchken solve the problem of non-smoothness by forcing the barrier to coincide with a layer of nodes. As discussed earlier, the problem with these approaches is that as the asset price gets close to the barrier, the number of time steps needed to value this option goes to infinity. This feature renders these models difficult to apply under these circumstances. ${ }^{22}$

In Cheuk and Vorst, the drift of each trinomial step can vary. When the spot price is close to the barrier, the drift can be adjusted to ensure a reasonably large step-size in the

\footnotetext{
${ }^{21}$ Gao (1996) discusses the pricing errors from the lattice model. He also shows that non-constant time steps can alleviate the problem only partially.

${ }^{22}$ Extrapolation methods may be helpful in this case, provided the individual elements in the sequence used for extrapolation (i.e. the option prices) can be computed with reasonable accuracy.
} 
price dimension, which is inversely related to the number of time periods required in the lattice. As a result, the convergence can be improved compared to the Ritchken method. However, for some range of parameter values with a fixed number of time steps, the CheukVorst scheme can produce negative probabilities and significant pricing errors, especially for long-term contracts with high volatility. ${ }^{23}$ Also, it remains to be shown how to compute hedge ratios using the method when the spot price is near the barrier, because step-sizes in price around the spot price are non-uniform.

The adaptive-mesh method developed by Gao solves the "near-barrier" problem by using a finer mesh around the barrier while maintaining a coarse structure in other places. It still suffers from the problem that the number of time steps goes to infinity as the asset price and the barrier get close to each other, although this happens only near the boundary in the time-price space, as opposed to everywhere in the restricted binomial/trinomial models. In contrast, as shown below, this sensitivity problem can be completely eliminated by using the analytic method developed here.

For a given method, the accuracy is measured by the deviation from a benchmark, more specifically by the root of the mean squared error (RMSE) or the root of the mean squared relative error (RMSRE). The benchmark is chosen to be the results from the Ritchken method with at least ten thousand time steps. ${ }^{24}$ The efficiency is measured by the CPU time required to compute option prices or hedge ratios for a given set of contracts. We choose two sets of contracts for comparison. Each set consists of forty-eight contracts that have different values of the underlying asset price $S_{t}$ at valuation date $t$, the time-toexpiration $T-t$, and the volatility parameter $\sigma$. The barrier level $H$ and the strike value $K$ are fixed at 50 and 45, respectively. The risk-free rate $r$ is chosen to be 0.0488. In Set I, we choose $S_{t}=(40,42.5,45,47.5), T-t=(0.25,0.5,0.75,1.0)$, and $\sigma=(0.2,0.3,0.4)$. As a result, the set of contracts include out-of-the-money, at-the-money, and in-the-money options. Set II is similar to Set I, except that those contracts with $S_{t}=47.5$ are replaced by contracts with $S_{t}=49.5$. The reason for this choice is to include contracts with $S_{t}$ very close to the barrier. This is the case where the existence of a barrier matters most.

Table 1 summarizes the statistics for option prices for the decomposition method and the trinomial methods in relation to the benchmark (the Ritchken (1995) method with at least ten thousand time-steps). Specifically, three schemes for implementing the decomposition method are included (c.f. Appendix V for details): (a) using a step-function to approximate

\footnotetext{
${ }^{23}$ For instance, consider a contract with $S_{t}=49.9, H=50, K=45, T-t=5$ (yr), and $\sigma=0.4$, whose true price is "very" close to 0.0634. Our implementation shows that the put value using the Cheuk-Vorst scheme equals 0.09 with $N=100,0.0640$ with $N=1,000$, and 0.0634 with $N=10,000$ respectively. Negative probabilities occur in all three cases.

${ }^{24}$ In the Ritchken method, the number of time steps cannot be chosen arbitrarily, due to the restriction that the barrier has to coincide with a node [see Ritchken (1995) for details]. In our implementation, the number of time steps used for contract Set I (to be specified below) is between 10,027 and 11,677, and for contract Set II (to be specified below) is between 10, 027 and 21, 385 .
} 
the exercise boundary combined with a 4-point Richardson extrapolation; (b) using a threepiece exponential function to approximate the exercise boundary without extrapolation; and (c) using a three-piece exponential function to approximate the exercise boundary combined with a 3-point Richardson extrapolation. For the trinomial methods, the Ritchken (1995) scheme (with a minimum 50 time steps in the trinomial tree) ${ }^{25}$ and the Cheuk-Vorst (1996) method (with the number of time steps $N=100$ ) are included for comparison. As can be seen, "penny" accuracy can be achieved in almost all cases for the values of the option.

Table 2 reports the statistics for hedge ratios for the Ritchken method and the three analytical approximations. Like the prices, the hedge ratios are within 0.01 of the benchmark in almost every case. The key issue, therefore is one of computational efficiency, given a level of accuracy.

Table 3 summarizes numerical results of the accuracy and speed of computation for option prices and deltas of our formulae (11) and (14) using the Ritchken method, the Cheuk-Vorst method, and the three approximation schemes mentioned above for the two sets of contracts. Columns 2 and 3 list the results for the contracts in Set I and columns 4 and 5 for the contracts in Set II. The results for the RMSE and RMSRE for all five methods are shown in the table, respectively. The CPU-times, the amount of time required (on a Sun Ultra 1 workstation) to compute the option prices or the delta values for all the forty-eight contracts in each set, are also presented in the table.

One can see from Table 3 that the errors from all the five methods are small under either of the two measures - RMSE and RMSRE - for both sets of contracts. The 3-step MPE with Richardson extrapolation clearly dominates the other methods in terms of accuracy.

Regarding speed, one can see from Table 3 that the step-function approximation is the fastest among the five methods. Also, except for the Ritchken method, the CPUtime required for Set II that includes the contracts with $S_{t}=49.5$ very close to the barrier $(H=50)$ is basically the same as that for set I. The Ritchken method is strongly dominated by the analytical approximation methods. This indicates that the quasi-analytic method can deal efficiently with the case in which the underlying price is very close to the barrier. As mentioned earlier, the reason is that the optimal exercise boundary, the sufficient input function of the valuation formula, is independent of the current underlying price. As a result, the problem of the underlying price being too close to the barrier is completely avoided in our approach.

To see how sensitive the three analytical approximation methods are to the magnitude of an option's American premium, we break up each set of contracts into two groups, namely a low-premium group and a high-premium one, and look at the performance of the three schemes within each group. Here, high-premium contracts within each set are those whose premia (the difference between the benchmark American and European option

\footnotetext{
${ }^{25}$ In our implementation of the Ritchken model, the number of time steps used is between 53 and 183 for Set I, and between 55 and 4,753 for Set II.
} 
values) ranked in the top half of the group, whereas low-premium contracts within each set are those whose premia ranked in the bottom half. Table 4 reports a summary of the results from the analytic methods between low- and high-premium contracts. Panels (a) and (b) summarize, respectively, the results for low- and high-premium options within each set of contracts. Columns 2 and 3 show the numerical results of option prices and delta values for contract set I. Columns 4 and 5 show the numerical results of option prices and delta values for contract set II. One can see from the table that the step-function scheme basically has lower RMSE but higher RMSRE in low-premium contracts than in high-premium contracts. Roughly, the same pattern holds for the 3-step MPE without extrapolation. The 3-step MPE extrapolation scheme has both lower RMSE and RMSRE in low-premium contracts than in high-premium contracts. These results seem to indicate that the 3-step MPE extrapolation scheme is the most sensitive to the premium among the analytical approximation methods. However, the errors from all three methods are small for both low- and high-premium contracts.

Overall, among the methods considered here, the 3-step MPE without Richardson extrapolation seems to provide the best balance between accuracy and computational efficiency. In summary, our numerical experiments show that the quasi-analytic pricing formula (11) is both accurate and efficient, and dominates the existing lattice methods. In particular, its performance is robust in the sense that both the accuracy and the efficiency are not sensitive to either the American option premium, or to the the distance between the underlying price and the barrier.

\section{Conclusion}

Non-standard or exotic options are in wide-spread use today in global financial markets. Increasingly, over-the-counter options on many assets including equities, fixed income securities, foreign exchange and commodities have non-standard characteristics, such as the "knock-out"/"knock-in" feature, and the averaging of the price of the underlying asset, among others. Often, due to the lack of liquid secondary markets for such products, in view of their custom-designed nature, an optimal exercise or American-style feature is incorporated into the design of the contract. It is well-known that, even for standard options, the American feature causes problems for valuation and hedging, since there is no closedform solution for the prices and hedge parameters, in general. Therefore, most models of American option valuation and hedging are implemented using numerical procedures. This problem is further compounded for non-standard American options.

It is understood that the use of numerical approaches for valuation and hedging of derivatives does have limitations. One is that almost all the available methods are based on a lattice or grid and the accuracy of the results obtained is limited by the fineness

of the grid. For exotic options such as barrier options, whose values are very sensitive to 
even minor perturbations in the parameters, the errors due to inappropriate lattices may be substantially large, and the computational time necessary to reduce these errors by choosing a finer grid size may be very intensive. In fast-moving markets, it is obviously essential to obtain reasonably accurate prices and hedge ratios fairly quickly. Another limitation is that even if one can come up with numerical methods that are fairly efficient and accurate, it is difficult to obtain an intuitive understanding of how the pricing and hedging works, in the absence of analytical results.

These problems make it desirable, whenever possible, to derive quasi-analytical models for non-standard American options. Our research shows that in many cases, such formulae can be derived, at least for some cases of exotic options, extending the work of Kim (1990), Jacka (1991) and Carr, Jarrow and Myneni (1992).

We are able to derive quasi-analytical formulae for the prices and hedge ratios in the case of barrier options (and "capped" options). The formulae are implemented using analytic approximations of the optimal exercise boundary and Richardson extrapolation. Our results indicate that our method is both accurate and efficient. In particular, the "near-boundary" sensitivity problem associated with using lattice methods is completely eliminated by using the technique developed here.

Our approach also indicates the advantage of studying the optimal exercise boundary when dealing with American options. We identify and exploit two key properties of the optimal exercise boundary - homogeneity in price parameters and translational invariance - for American options. In addition, some new put-call "symmetry" relations are also derived. These properties can be utilized to reduce repetitive computation of option prices and hedge ratios, and hence increase the efficiency of pricing and hedging American options. We present the details of our approach for American-style barrier options. The approach, based on the decomposition technique, can be applied to other non-standard American-style options such as look-back options and Asian options. ${ }^{26}$

\footnotetext{
${ }^{26}$ Recently, Hansen and Jorgensen (1998), applied the decomposition technique to the case of floatingstrike Asian Options.
} 


\section{Appendix I: Proof of Theorem 1}

Under suitable regularity conditions, we have that the discounted accumulative trading profits from holding an American option from time 0 to time $t$,

$$
\Lambda_{t}=G\left(S_{t}, t\right) e^{-r t}-G\left(S_{0}, 0\right)-\int_{0}^{t} \mathcal{D}^{s}\left[e^{-r u} G\left(S_{u}, u\right)\right] d u
$$

is a martingale under the risk-neutral measure [see for example Karatzas and Shreve (1991, p. 328)]. For instance, this holds if $G(\cdot)$ is the pricing function of standard American options. In the case of barrier options, the option pricing function $G\left(S_{t}, t\right)$ may not satisfy the usually assumed regularity conditions near the barrier. However, similar to standard European barrier-option pricing functions, $G\left(S_{t}, t\right)$ should satisfy those regularity conditions in the non-knock-out region where $M_{0}^{t}<H$ and which is what we focus on. Based on this argument, we claim that $\Lambda_{t}$ as defined above is a martingale when $G\left(S_{t}, t\right)$ represents the price of an "out-of-the-money" American "up-and-out" put option.

It follows that

$$
G\left(S_{0}, 0\right)=E_{0}\left[G\left(S_{T}, T\right) e^{-r T} I_{\left\{M_{0}^{T}<H\right\}}\right]-\int_{0}^{T} E_{0}\left[\mathcal{D}^{s}\left[e^{-r u} G\left(S_{u}, u\right)\right] I_{\left\{M_{0}^{u}<H\right\}}\right] d u .
$$

Using (2) and (5), we have

$$
\mathcal{D}^{s}\left[e^{-r u} G\left(S_{u}, u\right)\right]=e^{-r u}\left[\left(\mathcal{D}^{s}-r\right) h\left(S_{u}\right)\right] I_{\left\{\left(S_{u}, u\right) \in \mathcal{S}\right\}}=e^{-r u}\left(\delta S_{u}-r K\right) I_{\left\{\left(S_{u}, u\right) \in \mathcal{S}\right\}} .
$$

Substituting (35) into (34) yields (8) in Theorem 1. This completes the proof. 


\section{Appendix II: Proof of Theorem 2}

To simplify the notation, the subscript "uo" in $B_{\mathrm{uo}, t}$ is dropped in this appendix and the boundary point at $t$ is simply denoted by $B_{t}$.

Homogeneity: We prove this by induction in a discrete-time setting. The assertion is true for $B_{T}$ given the boundary condition

$$
B_{T}=\min [\min (r K / \delta, K), H] .
$$

Next consider $B_{T-1}$. Neglecting the early exercise premium, we have from (18)

$$
K-B_{T-1}=p_{\mathrm{uo}}\left(B_{T-1}, K, H\right),
$$

where $H$ has been explicitly specified as an argument. One can easily see from this equation that the assertion holds for $B_{T-1}$. Now suppose that it holds for $\left(B_{u}\right)_{u \geq t}$. Consider $B_{t-1}$. To simplify notation, let $\theta=(K, H)$. Again, we have from (18)

$$
K-B_{t-1}(\theta)=p_{\mathrm{uo}}\left(B_{t-1}(\theta), \theta\right)+f\left(B_{t-1}(\theta),\left\{B_{u}(\theta) ; u \geq t\right\}, \theta\right),
$$

where $f(\cdot)$ denotes the integral on the RHS of (18). Under the transformation

$$
\theta \rightarrow \alpha \theta \quad \forall \alpha \in \mathcal{R}_{++},
$$

the equation for the transformed boundary point $B_{t-1}(\alpha \theta)$ becomes

$$
\begin{aligned}
\alpha K-B_{t-1}(\alpha \theta) & =p_{\mathrm{uo}}\left(B_{t-1}(\alpha \theta), \alpha \theta\right)+f\left(B_{t-1}(\alpha \theta),\left\{B_{u}(\alpha \theta) ; u \geq t\right\}, \alpha \theta\right) \\
& =\alpha p_{\mathrm{uo}}\left(B_{t-1}(\alpha \theta) / \alpha, \theta\right)+f\left(B_{t-1}(\alpha \theta),\left\{\alpha B_{u}(\theta) ; u \geq t\right\}, \alpha \theta\right) \\
& =\alpha p_{\mathrm{uo}}\left(B_{t-1}(\alpha \theta) / \alpha, \theta\right)+\alpha f\left(B_{t-1}(\alpha \theta) / \alpha,\left\{B_{u}(\theta) ; u \geq t\right\}, \theta\right),
\end{aligned}
$$

where the homogeneity of $\left(B_{u}\right)_{u \geq t}$ has been used in the second equality. Dividing (37) by $\alpha$ on both sides, we have by definition ${ }^{27}$

$$
B_{t-1}(\alpha \theta) / \alpha=B_{t-1}(\theta),
$$

which says that $B_{t-1}(\theta)$ is homogeneous of degree one in $\theta$.

Translational Invariance in Time: Let $\left\{B_{t}(K, H, T) ; t \in[0, T]\right\}$ be the optimal exercise boundary of a contract with the expiration date $T$. It can be seen from (18) that

$$
B_{t}(K, H, T)=B_{t-u}(K, H, T-u) \quad \forall 0 \leq u \leq t \leq T
$$

Given fixed $T_{1}$ and $T_{2}$ where $T_{2}>T_{1}$, it follows that

$$
\begin{aligned}
B_{t}\left(K, H, T_{2}\right) & =B_{t-\left(T_{2}-T_{1}\right)}\left(K, H, T_{2}-\left(T_{2}-T_{1}\right)\right) \\
& =B_{t-\left(T_{2}-T_{1}\right)}\left(K, H, T_{1}\right) \quad \forall t \in\left[T_{2}-T_{1}, T_{2}\right]
\end{aligned}
$$

\footnotetext{
${ }^{27}$ The uniqueness of the boundary has been assumed implicitly.
} 
Monotonicity in Time: Differentiating (17) with respect to $t$ on both sides and using the fact that $\partial P_{\mathrm{uo}} / \partial t<0$ yields $\partial B_{t} / \partial t>0$.

Monotonicity in the Barrier Level: Given time $t$, it is obvious that an option price is an increasing function of the barrier level, i.e.

$$
\frac{\partial P_{\mathrm{uo}}\left(S_{t}, H, K\right)}{\partial H}>0
$$

Given that the optimal boundary condition satisfies

$$
K-B_{t}(H, K)=\lim _{S_{t} \downarrow B_{t}} P_{\mathrm{uo}}\left(S_{t}, H, K\right)
$$

one can take the partial derivative on the two sides, so that

$$
\frac{\partial B_{t}(H, K)}{\partial H}=-\lim _{S_{t} \downarrow B_{t}} \frac{\partial P_{\mathrm{uo}}\left(S_{t}, H, K\right)}{\partial H}<0 .
$$




\section{Appendix III: Proof of Theorem 3}

We prove the "put-call symmetry" for the case of the out-of-the-money "knock-out" option only. The case of the in-the-money "knock-out" can be analyzed in a similar fashion.

We define the notation first.

$$
\begin{aligned}
d_{1}(x, y, t, r, \delta) & =\frac{\ln (x / y)+\left(r-\delta+\sigma^{2} / 2\right) t}{\sigma \sqrt{t}} \\
d_{2}(x, y, t, r, \delta) & =\frac{\ln (x / y)+\left(r-\delta-\sigma^{2} / 2\right) t}{\sigma \sqrt{t}} \\
\lambda(r, \delta) & =\frac{r-\delta}{\sigma^{2}}+\frac{1}{2} \\
H^{\mathrm{P}} & =K S_{0} / H .
\end{aligned}
$$

To simplify the notation, we shall omit the subscript "do" or "uo" and use $B_{t}^{\mathrm{c}}$ and $B_{t}^{\mathrm{p}}$ to denote the optimal exercise boundary of a "down-and-out" call option and an "up-and-out" put option, respectively. Recall also that the superscript "o" denotes standard options. For instance, $c^{o}$ represents the price of a standard European call option. We know that the price of a "up-and-out" American put option $(K<H)$ is given by

$$
P_{\mathrm{uo}}\left(S_{0}, K, H, r, \delta\right)=p_{\mathrm{uo}}\left(S_{0}, K, H, r, \delta\right)+P_{\mathrm{uo}}^{p}\left(S_{0}, K, H, r, \delta\right)
$$

where

$$
\begin{aligned}
& p_{\text {uо }}\left(S_{0}, K, H, r, \delta\right)=p^{o}\left(S_{0}, K, r, \delta\right)-\left(H / S_{0}\right)^{2 \lambda(r, \delta)-2} p^{o}\left(H^{2} / S_{0}, K, r, \delta\right) \\
& P_{\text {uо }}^{p}\left(S_{0}, K, H, r, \delta\right)= \\
& \int_{0}^{T} e^{-r t}\left\{r K\left[N\left(-d_{2}\left(S_{0}, B_{t}, t, r, \delta\right)\right)-\left(H / S_{0}\right)^{2 \lambda(r, \delta)-2} N\left(-d_{2}\left(H^{2} / S_{0}, B_{t}, t, r, \delta\right)\right)\right]\right. \\
& \left.\quad-\delta S_{0}\left[N\left(-d_{1}\left(S_{0}, B_{t}, t, r, \delta\right)\right)-\left(H / S_{0}\right)^{2 \lambda(r, \delta)} N\left(-d_{1}\left(H^{2} / S_{0}, B_{t}, t, r, \delta\right)\right)\right]\right\} d t
\end{aligned}
$$

Similarly, the price of an "down-and-out" American call option $(K>H)$ is given by ${ }^{28}$

$$
C_{\mathrm{do}}\left(S_{0}, K, H, r, \delta\right)=c_{\mathrm{do}}\left(S_{0}, K, H, r, \delta\right)+C_{\mathrm{do}}^{p}\left(S_{0}, K, H, r, \delta\right)
$$

where

$$
\begin{aligned}
& c_{\mathrm{do}}\left(S_{0}, K, H, r, \delta\right)=c^{o}\left(S_{0}, K, r, \delta\right)-\left(H / S_{0}\right)^{2 \lambda(r, \delta)-2} c^{o}\left(H^{2} / S_{0}, K, r, \delta\right) \\
& C_{\mathrm{do}}^{p}\left(S_{0}, K, H, r, \delta\right)= \\
& \quad \int_{0}^{T} e^{-r t}\left\{\delta S_{0}\left[N\left(d_{1}\left(S_{0}, B_{t}, t, r, \delta\right)\right)-\left(H / S_{0}\right)^{2 \lambda(r, \delta)} N\left(d_{1}\left(H^{2} / S_{0}, B_{t}, t, r, \delta\right)\right)\right]\right. \\
& \left.\quad-r K\left[N\left(d_{2}\left(S_{0}, B_{t}, t, r, \delta\right)\right)-\left(H / S_{0}\right)^{2 \lambda(r, \delta)-2} N\left(d_{2}\left(H^{2} / S_{0}, B_{t}, t, r, \delta\right)\right)\right]\right\} d t .
\end{aligned}
$$

\footnotetext{
${ }^{28}$ See Rich (1994) for expressions for the option prices $c_{\text {do }}$ and $p_{\text {uo }}$. The early exercise (American) premium is derived by evaluating the expectation in equation (8), based on its equivalence to a long cash-or-nothing, and a short asset-or-nothing, knock-out option with a strike price equal to the value at the optimal boundary at time $t$. The option formula is readily available from those for a knock-out call and put. Detailed derivations are available upon request.
} 
Under the transformation

$$
S_{0} \rightarrow K, K \rightarrow S_{0}, \text { and } H \rightarrow K S_{0} / H
$$

it is easy to show, by direct substitution, that

$$
c_{\mathrm{do}}\left(S_{0}, K, H, r, \delta\right)=p_{\text {uo }}\left(K, S_{0}, K S_{0} / H, \delta, r\right) .
$$

This indicates that the put-call symmetry holds for the European part.

Next we will show that the premium part is also invariant under the transformation (44). Notice that under this transformation, optimal exercise boundary $B_{t}^{\mathrm{p}}$ for the "up-and-out" put option should be replaced by $K S_{0} / B_{t}^{\mathrm{c}}$. It is easy to show that the premium part is indeed invariant with this substitution. As a result, to complete the proof, we only have to show that $K S_{0} / B_{t}^{c}$ is the optimal exercise boundary for the "up-and-out" put with the strike price $S_{0}$ and barrier $H^{\mathrm{p}}=K S_{0} / H$. Namely, we need to prove the following condition:

$$
B_{t}^{\mathrm{p}}\left(S_{0}, H^{\mathrm{p}}, \delta, r\right) B_{t}^{\mathrm{c}}(K, H, r, \delta)=K S_{0}
$$

We prove this by induction. Consider $t=T$ first. Since $K>H$ implies $H^{\mathrm{p}}=K S_{0} / H>$ $S_{0}$, we know the optimal boundary for the put and the call at maturity is

$$
B_{T}^{\mathrm{p}}=\min \left(\delta S_{0} / r, S_{0}\right) \text { and } B_{T}^{\mathrm{c}}=\max (r K / \delta, K) .
$$

So the condition (45) is satisfied at the maturity.

Suppose now that the condition (45) holds at time $t+1$. Consider time $t$. Given that $B_{t}^{\mathrm{c}}$ is the optimal boundary at $t$ for the "down-and-out" call $C_{\mathrm{do}}\left(S_{0}, K, H, r, \delta\right)$, our goal is to show that $K S_{0} / B_{t}^{c}$ is the optimal boundary at $t$ for the "up-and-out" put $P_{\text {uo }}\left(K, S_{0}, K S_{0} / H, \delta, r\right)$. For the call option, the optimal boundary satisfies

$$
\begin{aligned}
B_{t}^{\mathrm{c}}- & K=c_{\mathrm{do}}^{o}\left(B_{t}^{\mathrm{c}}, K, H, r, \delta\right)+\int_{t}^{T} e^{-r(s-t)} \\
& \left\{\delta B_{t}^{\mathrm{c}}\left[N\left(d_{1}\left(B_{t}^{\mathrm{c}}, B_{s}^{\mathrm{c}}, s-t, r, \delta\right)\right)-\left(H / B_{t}^{\mathrm{c}}\right)^{2 \lambda(r, \delta)} N\left(d_{1}\left(H^{2} / B_{t}^{\mathrm{c}}, B_{s}^{\mathrm{c}}, s-t, r, \delta\right)\right)\right]\right. \\
& \left.-r K\left[N\left(d_{2}\left(B_{t}^{\mathrm{c}}, B_{s}^{\mathrm{c}}, s-t, r, \delta\right)\right)-\left(H / B_{t}^{\mathrm{c}}\right)^{2 \lambda(r, \delta)-2} N\left(d_{2}\left(H^{2} / B_{t}^{\mathrm{c}}, B_{s}^{\mathrm{c}}, s-t, r, \delta\right)\right)\right]\right\} d s .
\end{aligned}
$$

Applying the put-call symmetry condition to the European part of the above equation, we know

$$
c_{\mathrm{do}}^{o}\left(B_{t}^{\mathrm{c}}, K, H, r, \delta\right)=p_{\mathrm{uo}}^{o}\left(K, B_{t}^{\mathrm{c}}, K B_{t}^{\mathrm{c}} / H, \delta, r\right)
$$

From the homogeneity condition we know further that

$$
c_{\mathrm{do}}^{o}\left(B_{t}^{\mathrm{c}}, K, H, r, \delta\right)=\frac{B_{t}^{\mathrm{c}}}{S_{0}} p_{\mathrm{uo}}^{o}\left(K S_{0} / B_{t}^{\mathrm{c}}, S_{0}, K S_{0} / H, \delta, r\right) .
$$


Define $B_{t}^{\prime} \equiv K S_{0} / B_{t}^{\mathrm{c}}$. Our goal is to show that $B_{t}^{\mathrm{p}}=B_{t}^{\prime}$. Substituting (47) into (46) and then multiplying both sides of (46) by $S_{0} / B_{t}^{\mathrm{c}}$, we have

$$
\begin{aligned}
S_{0}- & B_{t}^{\prime}=p_{\mathrm{uo}}^{o}\left(B_{t}^{\prime}, S, K S_{0} / H, \delta, r\right)+\int_{t}^{T} e^{-r(s-t)} \\
& \left\{\delta S_{0}\left[N\left(d_{1}\left(B_{t}^{\mathrm{c}}, B_{s}^{\mathrm{c}}, s-t, r, \delta\right)\right)-\left(H / B_{t}^{\mathrm{c}}\right)^{2 \lambda(r, \delta)} N\left(d_{1}\left(H^{2} / B_{t}^{\mathrm{c}}, B_{s}^{\mathrm{c}}, s-t, r, \delta\right)\right)\right]\right. \\
& \left.-r B_{t}^{\prime}\left[N\left(d_{2}\left(B_{t}^{\mathrm{c}}, B_{s}^{\mathrm{c}}, s-t, r, \delta\right)\right)-\left(H / B_{t}^{\mathrm{c}}\right)^{2 \lambda(r, \delta)-2} N\left(d_{2}\left(H^{2} / B_{t}^{\mathrm{c}}, B_{s}^{\mathrm{c}}, s-t, r, \delta\right)\right)\right]\right\} d s .
\end{aligned}
$$

From the assumption about induction, we know that $B_{s}^{\mathrm{c}}=K S_{0} / B_{s}^{\mathrm{p}}$ for $s>t$, then

$$
\ln \frac{B_{t}^{\mathrm{c}}}{B_{s}^{\mathrm{c}}}=\ln \frac{B_{t}^{\mathrm{c}}}{K S_{0} / B_{s}^{\mathrm{p}}}=-\ln \frac{K S_{0} / B_{t}^{\mathrm{c}}}{B_{s}^{\mathrm{p}}}
$$

and

$$
\ln \frac{H^{2} / B_{t}^{\mathrm{c}}}{B_{s}^{\mathrm{c}}}=\ln \frac{H^{2} / B_{t}^{\mathrm{c}}}{K S_{0} / B_{s}^{\mathrm{p}}}=-\ln \frac{\left(H^{\mathrm{p}}\right)^{2} / B_{t}^{\prime}}{B_{s}^{\mathrm{p}}}
$$

Then

$$
\begin{aligned}
d_{1}\left(B_{t}^{\mathrm{c}}, B_{s}^{\mathrm{c}}, s-t, r, \delta\right) & =-d_{2}\left(B_{t}^{\prime}, B_{s}^{\mathrm{p}}, s-t, \delta, r\right), \\
d_{1}\left(H^{2} / B_{t}^{\mathrm{c}}, B_{s}^{\mathrm{c}}, s-t, r, \delta\right) & =-d_{2}\left(\left(H^{\mathrm{p}}\right)^{2} / B_{t}^{\prime}, B_{s}^{\mathrm{p}}, s-t, \delta, r\right), \\
d_{2}\left(B_{t}^{\mathrm{c}}, B_{s}^{\mathrm{c}}, s-t, r, \delta\right) & =-d_{1}\left(B_{t}^{\prime}, B_{s}^{\mathrm{p}}, s-t, \delta, r\right), \\
d_{2}\left(H^{2} / B_{t}^{\mathrm{c}}, B_{s}^{\mathrm{c}}, s-t, r, \delta\right) & =-d_{1}\left(\left(H^{\mathrm{p}}\right)^{2} / B_{t}^{\prime}, B_{s}^{\mathrm{p}}, s-t, \delta, r\right) .
\end{aligned}
$$

It follows that (48) becomes

$$
\begin{aligned}
S_{0}- & B_{t}^{\prime}=P_{\mathrm{uo}}^{o}\left(B_{t}^{\prime}, S, H^{\mathrm{p}}, \delta, r\right)+\int_{t}^{T} e^{-r(s-t)} \\
& \left\{\delta S_{0}\left[N\left(-d_{2}\left(B_{t}^{\prime}, B_{s}^{\mathrm{p}}, s-t, r, \delta\right)\right)-\left(H^{\mathrm{p}} / B_{t}^{\prime}\right)^{2 \lambda(\delta, r)-2} N\left(-d_{2}\left(\left(H^{\mathrm{p}}\right)^{2} / B_{t}^{\prime}, B_{s}^{\mathrm{p}}, s-t, r, \delta\right)\right)\right]\right. \\
& \left.-r B_{t}^{\prime}\left[N\left(-d_{1}\left(B_{t}^{\prime}, B_{s}^{\mathrm{p}}, s-t, r, \delta\right)\right)-\left(H^{\mathrm{p}} / B_{t}^{\prime}\right)^{2 \lambda(\delta, r)} N\left(-d_{1}\left(\left(H^{\mathrm{p}}\right)^{2} / B_{t}^{\prime}, B_{s}^{\mathrm{p}}, s-t, r, \delta\right)\right)\right]\right\} d s .
\end{aligned}
$$

This equation is identical to the optimal boundary equation for the "up-and-out" put option $P_{\mathrm{uo}}\left(K, S_{0}, K S_{0} / H, \delta, r\right)$ at time $t$ and this proves (28). Hence,

$$
B_{t}^{\mathrm{p}}=B_{t}^{\prime}=K S_{0} / B_{t}^{\mathrm{c}}
$$

is the solution of the equation. As a result, it follows from (45) that

$$
B_{\mathrm{do}, t}^{\mathrm{c}}(K, H, r, \delta)=\frac{S_{0} K}{B_{\mathrm{uo}, t}^{\mathrm{p}}\left(S_{0}, S_{0} K / H, \delta, r\right)}=\frac{K^{2}}{B_{\mathrm{uo}, t}^{\mathrm{p}}\left(K, K^{2} / H, \delta, r\right)},
$$

where the homogeneity of $B_{\mathrm{uo}, t}^{\mathrm{p}}$ in prices has been used in the last equality. This completes the proof. 


\section{Appendix IV: Proof of Theorem 4}

We prove Theorem 4 by contradiction. Suppose at a given time $t \in[0, T]$ with price $S_{t}$, it is optimal to continue, i.e., $P_{u o}\left(S_{t}, K\right)>K-S_{t}$. Consider a portfolio consisting of an American "up-and-out" put short, cash $K$ in the money market, and one share of the risky asset short. Constructing such a portfolio yields a net cash inflow of $P_{u o}(S, K)-(K-S)>0$ at time $t$.

Case a): The path of $S_{t}$ hits the barrier $H$ at time $\tau_{H}$, where $t<\tau_{H} \leq T$, before touching the exercise boundary. In this case, the option is knocked out. One can cover the short asset at $\operatorname{cost} H$, and at time $\tau_{H}$ realize a profit of $K e^{r\left(\tau_{H}-t\right)}-H>0$ (since $K>H$ by the assumption that the up-and-out put option is in-the-money).

Case b): The path of $S_{t}$ hits the exercise boundary at time $\tau_{E}$, where $t<\tau_{E}<T$, before crossing the barrier. In this case, the option is exercised. One can pay out the strike price $K$ from the money market account and get the underlying asset which can then be used to cover the short position. The net result at $\tau_{E}$ is a profit of $K e^{r\left(\tau_{E}-t\right)}-K>0$.

Case c): The path of $S_{t}$ hits neither the exercise boundary nor the barrier by $T^{-}$. This implies that $S_{T^{-}}<H<K$. By continuity, $S_{T}<H<K$. As a result, the option will be exercised at $T$. The net result at $T$ is a profit of $K e^{r(T-t)}-K>0$.

Thus, in all three cases, the position results in risk-free cash inflows at both time $t$ and later. Since this is against the no-arbitrage principle, the option must be exercised at some time $t^{\prime} \in[0, T]$. Therefore, it must be optimal to stop, i.e., to exercise the put option before the expiration date. 


\section{Appendix V: Approximations of the Optimal Exercise Bound- ary}

In this appendix, we discuss in detail how to implement the quasi-analytic formula (11). We focus on the multi-piece exponential (MPE) approximation scheme. The step-function approximation is a special case of the MPE approximation.

The MPE method is an extension of Ju's (1998) method for American options to American barrier options. Under this scheme, multiple exponential functions are used to approximate the optimal exercise boundary, each of which is defined by two variables which are determined by the continuity and smooth-pasting conditions. The advantage of using an exponential boundary is that the integrals representing the American premium can be computed analytically.

To simplify notation, the exercise boundary point at time $t$ is denoted by $B_{t}$ rather than $B_{\mathrm{uo}, t}^{p}$ in this appendix. Recall from (13) that the American option premium over some interval $\left[t_{1}, t_{2}\right]$ with $0 \leq t_{1}<t_{2} \leq T$ is given by

$$
P_{\mathrm{uo}}^{p}\left(S_{t_{1}}, t_{1}, t_{2}\right)=\int_{t_{1}}^{t_{2}} e^{-r t} r K\left[N\left(-d_{2}\left(S_{t_{1}}, B_{t}, t\right)\right)-\left(H / S_{t_{1}}\right)^{2 \lambda-2} N\left(-d_{2}\left(H^{2} / S_{t_{1}}, B_{t}, t\right)\right)\right] d t
$$

Assume $B_{t}=B e^{b t} \forall t \in\left[t_{1}, t_{2}\right]$ where parameters $B$ and $b$ are to be determined later. Under this approximation, the premium becomes

$$
P_{\mathrm{uo}}^{p}\left(S_{t_{1}}, K, B, b, t_{1}, t_{2}\right) \approx K I\left(S_{t_{1}}, B, b, t_{1}, t_{2}\right)-K\left(H / S_{t_{1}}\right)^{2 \lambda-2} I\left(H^{2} / S_{t_{1}}, B, b, t_{1}, t_{2}\right)
$$

where, integrating by parts,

$$
\begin{aligned}
I\left(S, B, b, t_{1}, t_{2}\right) \equiv & \int_{t_{1}}^{t_{2}} r N\left(-d_{2}\left(S, B e^{b t}, t\right)\right) e^{-r t} d t \\
= & e^{-r t_{1}} N\left(x t_{1}^{1 / 2}+y t_{1}^{-1 / 2}\right)-e^{-r t_{2}} N\left(x t_{2}^{1 / 2}+y t_{2}^{-1 / 2}\right) \\
& +\frac{1}{2}\left(\frac{x}{z}+1\right) e^{y(z-x)}\left[N\left(z t_{2}^{1 / 2}+y t_{2}^{-1 / 2}\right)-N\left(z t_{1}^{1 / 2}+y t_{1}^{-1 / 2}\right)\right] \\
& +\frac{1}{2}\left(\frac{x}{z}-1\right) e^{-y(z+x)}\left[N\left(z t_{2}^{1 / 2}-y t_{2}^{-1 / 2}\right)-N\left(z t_{1}^{1 / 2}-y t_{1}^{-1 / 2}\right)\right]
\end{aligned}
$$

with $x=-\left(r-b-\sigma^{2} / 2\right), y=-\ln (S / B) / \sigma$, and $z=\sqrt{x^{2}+2 r}$. Eq.(50) provides an analytical approximation of the American premium over $\left[t_{1}, t_{2}\right]$. An approximation of the premium (and hence the option price) over $[0, T]$ can then be obtained by repeating the above procedure for each element of a partition of the interval $[0, T]$.

Suppose the optimal exercise boundary is to be approximated by $N$ pieces of exponential functions. Let $B_{i}^{N} e^{b_{i}^{N} t}$ be the $i$ th exponential function with $B_{0}=B_{1}^{N}$. The boundary (and hence the option price and hedge ratios) is then specified by the set of parameters $\left(B_{i}^{N}, b_{i}^{N}\right)_{1 \leq i \leq N}$. Note that $\left(B_{i}^{N}, b_{i}^{N}=0\right)_{1 \leq i \leq N}$ defines the step-function approximation. 
Given the boundary, the option price can be determined as follows:

$$
P_{\mathrm{uo}}\left(S_{0}, 0, T\right) \approx\left\{\begin{array}{lll}
p_{\mathrm{uo}}\left(S_{0}, K, T\right)+\sum_{i=1}^{N} K I\left(S_{0}, B_{i}^{N}, b_{i}^{N}, \frac{i-1}{N} T, \frac{i}{N} T\right) & \\
\left.-\sum_{i=1}^{N}\left(\frac{H}{S_{0}}\right)^{2 \lambda-2} K I\left(\frac{H^{2}}{S_{0}}, B_{i}^{N}, b_{i}^{N}, \frac{i-1}{N} T, \frac{i}{N} T\right)\right) & \text { if } \quad S_{0}>B_{1}^{N} \\
K-S_{0} & \text { if } \quad S_{0} \leq B_{1}^{N} .
\end{array}\right.
$$

The option delta can be obtained by differentiating the above price w.r.t. the spot price. Namely,

$$
\begin{aligned}
& \frac{\partial P_{\mathrm{uo}}}{\partial S_{0}}\left(S_{0}, 0, T\right) \\
& \approx \begin{cases}\frac{\partial p_{\mathrm{uo}}}{\partial S}\left(S_{0}, K, T\right) & \\
& +\sum_{i=1}^{N} K I_{S}\left(S_{0}, B_{i}^{N}, b_{i}^{N}, \frac{i-1}{N} T, \frac{i}{N} T\right) \\
& \left.+\sum_{i=1}^{N}\left(\frac{H}{S_{0}}\right)^{2 \lambda} K I_{S}\left(\frac{H^{2}}{S_{0}}, B_{i}^{N}, b_{i}^{N}, \frac{i-1}{N} T, \frac{i}{N} T\right)\right) \\
& \left.+(2 \lambda-2) \sum_{i=1}^{N}\left(\frac{H}{S_{0}}\right)^{2 \lambda-2}\left(\frac{K}{S_{0}}\right) I\left(\frac{H^{2}}{S_{0}}, B_{i}^{N}, b_{i}^{N}, \frac{i-1}{N} T, \frac{i}{N} T\right)\right) \\
-1 & \text { if } \quad S_{0}>B_{1}^{N} \\
-1 & \text { if } \quad S_{0} \leq B_{1}^{N},\end{cases}
\end{aligned}
$$

where

$$
\begin{aligned}
I_{S}\left(S, B, b, t_{1}, t_{2}\right) & =-\frac{1}{\sigma S}\left\{e^{-r t_{1}} n\left(x t_{1}^{1 / 2}+y t_{1}^{-1 / 2}\right) t_{1}^{-1 / 2}-e^{-r t_{2}} n\left(x t_{2}^{1 / 2}+y t_{2}^{-1 / 2}\right) t_{2}^{-1 / 2}\right. \\
& +\frac{1}{2}\left(\frac{x}{z}+1\right) e^{y(z-x)}(z-x)\left[N\left(z t_{2}^{1 / 2}+y t_{2}^{-1 / 2}\right)-N\left(z t_{1}^{1 / 2}+y t_{1}^{-1 / 2}\right)\right] \\
& -\frac{1}{2}\left(\frac{x}{z}-1\right) e^{-y(z+x)}(z+x)\left[N\left(z t_{2}^{1 / 2}-y t_{2}^{-1 / 2}\right)-N\left(z t_{1}^{1 / 2}-y t_{1}^{-1 / 2}\right)\right] \\
& +\frac{1}{2}\left(\frac{x}{z}+1\right) e^{y(z-x)}\left[n\left(z t_{2}^{1 / 2}+y t_{2}^{-1 / 2}\right) t_{2}^{-1 / 2}-n\left(z t_{1}^{1 / 2}+y t_{1}^{-1 / 2}\right) t_{1}^{-1 / 2}\right] \\
& \left.-\frac{1}{2}\left(\frac{x}{z}-1\right) e^{-y(z+x)}\left[n\left(z t_{2}^{1 / 2}-y t_{2}^{-1 / 2}\right) t_{2}^{-1 / 2}-n\left(z t_{1}^{1 / 2}-y t_{1}^{-1 / 2}\right) t_{1}^{-1 / 2}\right]\right\}
\end{aligned}
$$

The remaining task is to determine $2 N$ parameters, $B_{i}^{N}, b_{i}^{N}, i=1,2, \cdots, N$. This can be done by using the recursive scheme discussed earlier (c.f. Sec. 3.1). In the case of stepfunctions, each of $\left(B_{i}^{N}\right)_{1 \leq i \leq N}$ can be obtained by solving numerically a nonlinear equation (the continuity condition). In the case of exponential functions, each pair of $\left(B_{i}^{N}, b_{i}^{N}\right)_{1 \leq i \leq N}$ has to be determined by simultaneously solving two nonlinear equations (the continuity and smooth-pasting equations). For instance, given $\left(B_{j}^{N}, b_{j}^{N}\right)_{j>i}$ at time $t=\frac{i-1}{N} T$ the pair of parameters $\left(B_{i}^{N}, b_{i}^{N}\right)$ can be obtained by solving the following two equations

$$
\begin{aligned}
K-B_{i}^{N} e^{b_{i}^{N} \frac{i-1}{N} T} & =p_{\text {uo }}\left(B_{i}^{N} e^{b_{i}^{N} \frac{i-1}{N} T}, K, \frac{N-i+1}{N} T\right) \\
& +\sum_{j=i}^{N} K I\left(B_{i}^{N} e^{b_{i}^{N} \frac{i-1}{N} T}, B_{j}^{N} e^{b_{j}^{N} \frac{i-1}{N} T}, b_{j}^{N}, \frac{j-i}{N} T, \frac{j-i+1}{N} T\right) \\
& -\sum_{j=i}^{N}\left(\frac{H}{B_{i}^{N} e^{b_{i}^{N} \frac{i-1}{N} T}}\right)^{2 \lambda-2} K I\left(\frac{H^{2}}{B_{i}^{N} e^{b_{i}^{N} \frac{i-1}{N}} T}, B_{j}^{N} e^{b_{j}^{N} \frac{i-1}{N} T}, b_{j}^{N}, \frac{j-i}{N} T, \frac{j-i+1}{N} T\right)
\end{aligned}
$$


and

$$
\begin{aligned}
& -1=-N\left(d_{1}\left(B_{i}^{N} e^{b_{i}^{N} \frac{i-1}{N} T}, K, \frac{N-i+1}{N} T\right)\right) \\
& +\sum_{j=i}^{N} K I_{S}\left(B_{i}^{N} e^{b_{i}^{N} \frac{i-1}{N} T}, B_{j}^{N} e^{b_{j}^{N} \frac{i-1}{N} T}, b_{i}^{N}, \frac{j-i}{N} T, \frac{j-i+1}{N} T\right) \\
& +\sum_{j=i}^{N}\left(\frac{H}{B_{i}^{N} e^{b_{i}^{N} \frac{i-1}{N} T}}\right)^{2 \lambda} K I_{S}\left(S=\frac{H^{2}}{B_{i}^{N} e^{b_{i}^{N} \frac{i-1}{N} T}}, B_{j}^{N} e^{b_{j}^{N} \frac{i-1}{N} T}, b_{i}^{N}, \frac{j-i}{N} T, \frac{j-i+1}{N} T\right) \\
& +(2 \lambda-2) \sum_{j=i}^{N}\left(\frac{H}{B_{i}^{N} e^{b_{i}^{N} \frac{i-1}{N} T}}\right)^{2 \lambda-2}\left(\frac{K}{S_{0}}\right) I\left(\frac{H^{2}}{B_{i}^{N} e^{b_{i}^{N} \frac{i-1}{N} T}}, B_{j}^{N} e^{b_{j}^{N} \frac{i-1}{N} T}, b_{i}^{N}, \frac{j-i}{N} T, \frac{j-i+1}{N} T\right)
\end{aligned}
$$

As mentioned earlier, estimates of $P_{\text {uo }}$ obtained using different values of $N$ can be used to extrapolate the true option price. For instance, given the estimates $P^{(i)}$ corresponding to $N=i, i=1,2,3$, the following 3-point Richardson extrapolation can be used to compute $P_{\mathrm{uo}}$ :

$$
P_{\mathrm{uo}} \approx\left(9 P^{(3)}-8 P^{(2)}+P^{(1)}\right) / 2 .
$$




\section{References}

Barone-Adesi, G., and R. Whaley, 1987, "Efficient Analytic Approximation of American Option Values," Journal of Finance, 42, 301-320.

Black, F., and J. Cox, 1976, "Valuing Corporate Securities: Some Effects of Bond Indenture Provisions," Journal of Finance, 31, 351-367.

Black, F., and M. Scholes, 1973, "The Pricing of Options and Corporate Liabilities," Journal of Political Economy, 81, 637-59.

Boyle, P. and S. H. Lau, 1994, "Bumping Up against the Barrier with the Binomial Method," Journal of Derivatives, 2, 6-14.

Boyle, P. and S. Turnbull, 1989, "Pricing and Hedging Capped Options," Journal of Futures Markets, 9, 41-54.

Brealey, R. A., S. D. Hodges, and M.J.P. Selby, 1983, “The Risk of Bank-Loan Portfolios," in Option Pricing, ed., M. Brenner, Lexington, Mass.:D.C. Heath, 153-182.

Breen, R., 1991, "The Accelerated Binomial Option Pricing Model," Journal of Financial and Quantitative Analysis, 26, 153-164.

Broadie, M., and J. Detemple, 1995, "American Capped Call Options on Dividend-Paying Assets," Review of Financial Studies, 8, 161-191.

Broadie, M., and J. Detemple, 1996, "American Option Valuation, New Bounds, Approximations, and a Comparison of Existing Methods," Review of Financial Studies, 11, 1211-1250.

Broadie, M., and J. Detemple, 1997, "Valuation of American Options on Multiple Assets," Mathematical Finance, 7, 241-286.

Bunch, D.S., and H. Johnson, 1992, "A Simple and Numerically Efficient Valuation Method for American Puts Using a Modified Geske-Johnson Approach," Journal of Finance, 47, 809-816.

Carr, P., 1998, "Randomization and the American Put," Review of Financial Studies, $11,597-626$.

Carr, P., K. Ellis, and V. Gupta, 1998, "Static Hedging of Exotic Options," Journal of Finance, 53, 1165-1190.

Carr, P., and D. Faguet, 1995, "Fast Accurate Valuation of American Options," working paper, Cornell University. 
Carr, P., R. Jarrow and R. Myneni, 1992, "Alternative Characterizations of American Put Options," Mathematical Finance, 2, 87-106.

Chesney, M., and R. Gibson, 1993, "State Space Symmetry and Two-Factor Option Pricing Models," Advances in Futures and Options Research, 8, 85-112.

Cheuk, T.H.F., and T.C.F. Vorst, 1996, "Complex Barrier Options," Journal of Derivatives, 4, 8-22.

Cox, D.R., and H.D. Miller, 1965, "The Theory of Stochastic Processes," Chapman and Hall, London.

Cox, J.C., and S.A. Ross, 1976, "The Valuation of Options for Alternative Stochastic Processes," Journal of Financial Economics, 3, 145-166.

Derman, E., D. Ergener and I. Kani, 1995, "Static Options Replication," Journal of Derivatives, 2, 78-95.

Duffie, D., 1992, Dynamic Asset Pricing Theory. Princeton University Press, Princeton, NJ.

Gao, B., 1996, "Essays in Efficient Option Pricing," Ph.D Dissertation, New York University.

Geske, R., and H.E. Johnson, 1984, "The American Put Options Valued Analytically," Journal of Finance, 39, 1511-1524.

Hansen, A., and P. Jorgensen, 1998, "Analytical Valuation of American-style Asian Options," working paper, University of Aarhus.

Harrison, J.M., and D.M. Kreps, 1979, "Martingales and Arbitrage in Multiperiod Securities Markets," Journal of Economic Theory, 20, 381-408.

Harrison, J.M., and S. Pliska, 1981, "Martingale and Stochastic Integrals in the Theory of Continuous Trading," Stochastic Processes and Their Applications, 11, 215-260.

Ho, T.S., R.C. Stapleton, and M.G. Subrahmanyam, 1994, "A Simple Technique for the Valuation and Hedging of American Options," Journal of Derivatives, 2, 55-75.

Ho, T.S., R.C. Stapleton, and M.G. Subrahmanyam, 1997a, "The Valuation of American Options with Stochastic Interest Rates: A Generalization of the Geske-Johnson Technique," Journal of Finance, 52, 827-840.

Ho, T.S., R.C. Stapleton, and M.G. Subrahmanyam, 1997b, "The Valuation of AmericanStyle Options on Bonds," Journal of Banking and Finance, 21, 1487-1513. 
Hsu, H., 1997, "Surprised Parties," Risk, 10, 27-29.

Huang, J.Z., M.G. Subrahmanyam, and G. G. Yu, 1996, "Pricing and Hedging American Options: A Recursive Integration Method," Review of Financial Studies, 9, 277-300.

Jacka, S., 1991, "Optimal Stopping and the American Put," Mathematical Finance, 1, $1-14$.

Joubert, and C. Rogers, 1997, "Fast, Accurate, and Inelegant Valuation of American Options," in Numerical Methods in Finance, eds. C. Rogers and Talay, Cambridge University Press, 88-92.

Ju, N., 1998, "Pricing an American Option by Approximating its Early Exercise Boundary As a Piece-Wise Exponential Function," Review of Financial Studies, 11, 627-646.

Karatzas, I., and S. Shreve, 1991, Brownian Motion and Stochastic Calculus, 2nd. ed., Springer-Verlag, New York.

Kim, I.J., 1990, "The Analytic Valuation of American Options," Review of Financial Studies, 3, 547-572.

MacMillan, L.W., 1986, "An Analytic Approximation for the American Put Price," $A d$ vances in Futures and Options Research, 1, 119-139.

McDonald, R., and M. Schroder, 1998, "A Parity Result for American Options," Journal of Computational Finance, 1, 5-13.

McKean, H.P. Jr., 1965, “Appendix: A Free Boundary Problem for the Heating Function Arising from a Problem in Mathematical Economics," Industrial Management Review, $6,32-39$.

Merton, R.C., 1973, "Theory of Rational Option Pricing," Bell Journal of Economics and Management Science, 4, 141-183.

Merton, R.C., 1990, Continuous-Time Finance, Blackwell, Cambridge, MA.

Omberg, E., 1987, "The Valuation of American Put Options with Exponential Exercise Policies," Advances in Futures and Options Research, 2, 117-142.

Rich, D., 1994, "The Mathematical Foundations of Barrier Option-Pricing Theory", Advances in Futures and Options Research, 7, 267-311.

Ritchken, P., 1995, "On Pricing Barrier Options," Journal of Derivatives, 3, 19-28.

Rogers, L.C.G and E.S. Stapleton, 1998, "Fast Accurate Binomial Pricing," Finance and Stochastics, 2, 3-17. 
Rubinstein, M. and E. Reiner, 1991, "Breaking Down the Barriers," RISK, 4, 28-35.

Schroder, M., 1989, "A Reduction Method Applicable to Compound Option Formulas," Management Science, 35, 823-827.

Schroder, M., 1997, "Changes of Numeraire for Pricing Futures, Forwards and Options," working paper, SUNY at Buffalo.

Selby, M.J.P., 1983, "The Application of Option Theory to the Evaluation of Risky Debt," Ph.D. Thesis, University of London.

Selby, M.J.P., and S.D. Hodges, 1987, "On the Evaluation of Compound Options," Management Science, 33, 347-355.

van Moerbeke, P., 1976, "On Optimal Stopping and Free Boundary Problems," Archive for Rational Mechanics and Analysis, 60, 101-148. 
Table 1: Prices of American "Up-and-Out" Put Options on Non-Dividend-Paying Stocks

( $K=\$ 45 ; H=\$ 50 ; r=4.88 \%)$

(a) Option Prices for Contract Set I

\begin{tabular}{|c|c|c|c|c|c|c|c|c|c|}
\hline $\begin{array}{l}(1) \\
\end{array}$ & $\overline{(2)}$ & $\begin{array}{l}(3) \\
\end{array}$ & $\overline{(4)}$ & $\overline{(5)}$ & $\overline{(76)}$ & $\overline{(7(7)}$ & $\begin{array}{l}(8) \\
\end{array}$ & $\begin{array}{l}(9) \\
\end{array}$ & 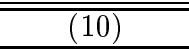 \\
\hline$S_{t}$ & $\sigma$ & $T-t$ & $\begin{array}{c}\text { European } \\
\text { price }\end{array}$ & $\begin{array}{l}\text { Bench- } \\
\text { mark }\end{array}$ & Ritchken & $\begin{array}{l}\text { Cheuk- } \\
\text { Vorst }\end{array}$ & $\begin{array}{c}\text { Step-Func } \\
\text { w/RE }\end{array}$ & $\begin{array}{l}\text { 3-Step } \\
\text { MPE }\end{array}$ & $\begin{array}{c}\text { 3-Step } \\
\mathrm{MPE} \text { w/ RE }\end{array}$ \\
\hline 40.0 & 0.2 & 0.25 & 4.7690 & 5.0357 & 5.0348 & 5.0351 & 5.0395 & 5.0353 & 5.0360 \\
\hline 40.0 & 0.2 & 0.50 & 4.7986 & 5.1881 & 5.1828 & 5.1874 & 5.1840 & 5.1869 & 5.1893 \\
\hline 40.0 & 0.2 & 0.75 & 4.7996 & 5.3083 & 5.3034 & 5.3084 & 5.3000 & 5.3067 & 5.3095 \\
\hline 40.0 & 0.2 & 1.00 & 4.7592 & 5.3861 & 5.3797 & 5.3870 & 5.3755 & 5.3843 & 5.3868 \\
\hline 42.5 & 0.2 & 0.25 & 2.8782 & 2.9958 & 2.9962 & 2.9972 & 2.9952 & 2.9945 & 2.9963 \\
\hline 42.5 & 0.2 & 0.50 & 3.1440 & 3.3476 & 3.3443 & 3.3490 & 3.3456 & 3.3456 & 3.3490 \\
\hline 42.5 & 0.2 & 0.75 & 3.2508 & 3.5379 & 3.5361 & 3.5399 & 3.5328 & 3.5357 & 3.5391 \\
\hline 42.5 & 0.2 & 1.00 & 3.2753 & 3.6446 & 3.6403 & 3.6466 & 3.6356 & 3.6423 & 3.6453 \\
\hline 45.0 & 0.2 & 0.25 & 1.4980 & 1.5445 & 1.5390 & 1.5470 & 1.5479 & 1.5430 & 1.5448 \\
\hline 45.0 & 0.2 & 0.50 & 1.8385 & 1.9375 & 1.9331 & 1.9391 & 1.9433 & 1.9357 & 1.9384 \\
\hline 45.0 & 0.2 & 0.75 & 1.9686 & 2.1197 & 2.1149 & 2.1217 & 2.1259 & 2.1178 & 2.1204 \\
\hline 45.0 & 0.2 & 1.00 & 2.0128 & 2.2151 & 2.2111 & 2.2180 & 2.2198 & 2.2132 & 2.2154 \\
\hline 47.5 & 0.2 & 0.25 & 0.6007 & 0.6159 & 0.6151 & 0.6185 & 0.6167 & 0.6150 & 0.6161 \\
\hline 47.5 & 0.2 & 0.50 & 0.8232 & 0.8625 & 0.8609 & 0.8636 & 0.8655 & 0.8614 & 0.8628 \\
\hline 47.5 & 0.2 & 0.75 & 0.9049 & 0.9686 & 0.9676 & 0.9706 & 0.9738 & 0.9675 & 0.9688 \\
\hline 47.5 & 0.2 & 1.00 & 0.9347 & 1.0223 & 1.0215 & 1.0252 & 1.0283 & 1.0213 & 1.0224 \\
\hline 40.0 & 0.3 & 0.25 & 5.3187 & 5.4639 & 5.4631 & 5.4658 & 5.4621 & 5.4624 & 5.4645 \\
\hline 40.0 & 0.3 & 0.50 & 5.6130 & 5.8526 & 5.8507 & 5.8541 & 5.8497 & 5.8502 & 5.8534 \\
\hline 40.0 & 0.3 & 0.75 & 5.7112 & 6.0453 & 6.0394 & 6.0470 & 6.0389 & 6.0427 & 6.0463 \\
\hline 40.0 & 0.3 & 1.00 & 5.7155 & 6.1455 & 6.1394 & 6.1471 & 6.1342 & 6.1431 & 6.1465 \\
\hline 42.5 & 0.3 & 0.25 & 3.5987 & 3.6798 & 3.6788 & 3.6831 & 3.6840 & 3.6780 & 3.6801 \\
\hline 42.5 & 0.3 & 0.50 & 3.9815 & 4.1314 & 4.1311 & 4.1341 & 4.1369 & 4.1290 & 4.1318 \\
\hline 42.5 & 0.3 & 0.75 & 4.1075 & 4.3255 & 4.3199 & 4.3277 & 4.3297 & 4.3232 & 4.3262 \\
\hline 42.5 & 0.3 & 1.00 & 4.1342 & 4.4213 & 4.4171 & 4.4244 & 4.4212 & 4.4191 & 4.4219 \\
\hline 45.0 & 0.3 & 0.25 & 2.1830 & 2.2250 & 2.2179 & 2.2287 & 2.2282 & 2.2235 & 2.2254 \\
\hline 45.0 & 0.3 & 0.50 & 2.5268 & 2.6135 & 2.6104 & 2.6151 & 2.6197 & 2.6117 & 2.6137 \\
\hline 45.0 & 0.3 & 0.75 & 2.6361 & 2.7668 & 2.7625 & 2.7699 & 2.7743 & 2.7650 & 2.7671 \\
\hline 45.0 & 0.3 & 1.00 & 2.6648 & 2.8399 & 2.8372 & 2.8417 & 2.8458 & 2.8383 & 2.8402 \\
\hline 47.5 & 0.3 & 0.25 & 1.0150 & 1.0327 & 1.0315 & 1.0350 & 1.0338 & 1.0318 & 1.0329 \\
\hline 47.5 & 0.3 & 0.50 & 1.2130 & 1.2524 & 1.2494 & 1.2533 & 1.2557 & 1.2514 & 1.2524 \\
\hline 47.5 & 0.3 & 0.75 & 1.2745 & 1.3352 & 1.3334 & 1.3367 & 1.3401 & 1.3342 & 1.3352 \\
\hline 47.5 & 0.3 & 1.00 & 1.2918 & 1.3739 & 1.3729 & 1.3760 & 1.3787 & 1.3731 & 1.3741 \\
\hline 40.0 & 0.4 & 0.25 & 5.8723 & 5.9773 & 5.9781 & 5.9796 & 5.9809 & 5.9754 & 5.9778 \\
\hline 40.0 & 0.4 & 0.50 & 6.2435 & 6.4285 & 6.4244 & 6.4310 & 6.4326 & 6.4260 & 6.4292 \\
\hline 40.0 & 0.4 & 0.75 & 6.3496 & 6.6162 & 6.6147 & 6.6207 & 6.6173 & 6.6137 & 6.6171 \\
\hline 40.0 & 0.4 & 1.00 & 6.3540 & 6.7054 & 6.7029 & 6.7077 & 6.7006 & 6.7032 & 6.7063 \\
\hline 42.5 & 0.4 & 0.25 & 4.1768 & 4.2424 & 4.2438 & 4.2455 & 4.2470 & 4.2406 & 4.2427 \\
\hline 42.5 & 0.4 & 0.50 & 4.5514 & 4.6759 & 4.6721 & 4.6782 & 4.6831 & 4.6737 & 4.6764 \\
\hline 42.5 & 0.4 & 0.75 & 4.6580 & 4.8422 & 4.8396 & 4.8451 & 4.8489 & 4.8400 & 4.8427 \\
\hline 42.5 & 0.4 & 1.00 & 4.6727 & 4.9188 & 4.9155 & 4.9218 & 4.9213 & 4.9169 & 4.9194 \\
\hline 45.0 & 0.4 & 0.25 & 2.6628 & 2.7007 & 2.6971 & 2.7042 & 2.7036 & 2.6993 & 2.7010 \\
\hline 45.0 & 0.4 & 0.50 & 2.9602 & 3.0368 & 3.0336 & 3.0383 & 3.0428 & 3.0352 & 3.0370 \\
\hline 45.0 & 0.4 & 0.75 & 3.0436 & 3.1591 & 3.1572 & 3.1620 & 3.1663 & 3.1576 & 3.1594 \\
\hline 45.0 & 0.4 & 1.00 & 3.0586 & 3.2145 & 3.2133 & 3.2179 & 3.2195 & 3.2131 & 3.2148 \\
\hline 47.5 & 0.4 & 0.25 & 1.2875 & 1.3049 & 1.3043 & 1.3081 & 1.3060 & 1.3041 & 1.3050 \\
\hline 47.5 & 0.4 & 0.50 & 1.4496 & 1.4859 & 1.4846 & 1.4875 & 1.4891 & 1.4851 & 1.4860 \\
\hline 47.5 & 0.4 & 0.75 & 1.4946 & 1.5500 & 1.5493 & 1.5528 & 1.5543 & 1.5492 & 1.5501 \\
\hline 47.5 & 0.4 & 1.00 & 1.5035 & 1.5787 & 1.5781 & 1.5822 & 1.5822 & 1.5780 & 1.5788 \\
\hline
\end{tabular}


(b) Option Prices for Contract Set II

\begin{tabular}{cccccccccc}
\hline \hline$(1)$ & $(2)$ & $(3)$ & $(4)$ & $(5)$ & $(6)$ & $(7)$ & $(8)$ & $(9)$ & $(10)$ \\
\hline & & & $\begin{array}{c}\text { European } \\
\text { price }\end{array}$ & $\begin{array}{c}\text { Bench- } \\
\text { mark }\end{array}$ & Ritchken & $\begin{array}{c}\text { Cheuk- } \\
\text { Vorst }\end{array}$ & $\begin{array}{c}\text { Step-Func } \\
\text { w/ RE }\end{array}$ & $\begin{array}{c}\text { 3-Step } \\
\text { MPE }\end{array}$ & $\begin{array}{c}\text { 3-Step } \\
\text { MPE w/ RE }\end{array}$ \\
\hline 49.5 & 0.2 & 0.25 & 0.1077 & 0.1103 & 0.1103 & 0.1107 & 0.1104 & 0.1101 & 0.1103 \\
49.5 & 0.2 & 0.50 & 0.1542 & 0.1613 & 0.1613 & 0.1619 & 0.1618 & 0.1611 & 0.1614 \\
49.5 & 0.2 & 0.75 & 0.1711 & 0.1828 & 0.1828 & 0.1836 & 0.1839 & 0.1826 & 0.1828 \\
49.5 & 0.2 & 1.00 & 0.1773 & 0.1936 & 0.1936 & 0.1945 & 0.1949 & 0.1934 & 0.1936 \\
\hline 49.5 & 0.3 & 0.25 & 0.1957 & 0.1990 & 0.1990 & 0.1999 & 0.1992 & 0.1988 & 0.1990 \\
49.5 & 0.3 & 0.50 & 0.2364 & 0.2439 & 0.2439 & 0.2449 & 0.2446 & 0.2437 & 0.2439 \\
49.5 & 0.3 & 0.75 & 0.2489 & 0.2606 & 0.2606 & 0.2619 & 0.2617 & 0.2604 & 0.2606 \\
49.5 & 0.3 & 1.00 & 0.2525 & 0.2684 & 0.2684 & 0.2700 & 0.2695 & 0.2682 & 0.2684 \\
\hline 49.5 & 0.4 & 0.25 & 0.2529 & 0.2563 & 0.2563 & 0.2574 & 0.2565 & 0.2561 & 0.2563 \\
49.5 & 0.4 & 0.50 & 0.2859 & 0.2930 & 0.2930 & 0.2944 & 0.2936 & 0.2928 & 0.2930 \\
49.5 & 0.4 & 0.75 & 0.2950 & 0.3059 & 0.3059 & 0.3076 & 0.3068 & 0.3057 & 0.3059 \\
49.5 & 0.4 & 1.00 & 0.2969 & 0.3117 & 0.3117 & 0.3137 & 0.3124 & 0.3115 & 0.3117 \\
\hline
\end{tabular}

Table 1 reports the values of American "up-and-out" put options on non-dividend-paying stocks for two sets of contracts computed using different methods. Set I includes 48 contracts, each of which has a different value of the parameter set $\left(S_{t}, T-t, \sigma\right)$. The domain of this parameter set is $S_{t}=(40,42.5,45,47.5)$, $T-t=(0.25,0.5,0.75,1.0)$, and $\sigma=(0.2,0.3,0.4)$. Set II is similar to set I, except that those contracts with $S_{t}=47.5$ are replaced by contracts with $S_{t}=49.5$. Panels (a) and (b) show the numerical results for contract set I and II, respectively. Columns 1 through 3 represent the values of the parameters, $S_{t}$ (the time- $t$ stock price), $\sigma$ (volatility), and $T-t$ (the time to expiration), respectively. Column 4 reports the European option values obtained using the analytic formula in (12). Columns 5 through 7 show the numerical results of option values from the Ritchken method with at least 10,000 time steps (the benchmark), the Ritchken method with at least 50 time steps, and the Cheuk-Vorst method with 100 time steps, respectively. Columns 8 through 10 show the numerical results from three analytic approximation techniques: the stepfunction scheme using a 4-point Richardson extrapolation, and the 3-step multi-piece exponential (MPE) approximation with and without Richardson extrapolation. 
Table 2: Deltas of American "Up-and-Out" Put Options on Non-Dividend-Paying Stocks

$$
(K=\$ 45 ; H=\$ 50 ; r=4.88 \%)
$$

(a) Option Deltas for Contract Set I

\begin{tabular}{|c|c|c|c|c|c|c|c|c|}
\hline$(1)$ & $(2)$ & $(3)$ & $(4)$ & $(5)$ & $(6)$ & $(7)$ & $(8)$ & $(9)$ \\
\hline$S_{t}$ & $\sigma$ & $T-t$ & $\begin{array}{c}\text { European } \\
\text { delta }\end{array}$ & $\begin{array}{c}\text { Bench- } \\
\text { mark }\end{array}$ & Ritchken & $\begin{array}{c}\text { Step-Func } \\
\text { w/ RE }\end{array}$ & $\begin{array}{c}\text { 3-Step MPE } \\
\text { w/o RE } \\
\end{array}$ & $\begin{array}{c}\text { 3-Step MPE } \\
\text { w/ RE } \\
\end{array}$ \\
\hline 40.0 & 0.2 & 0.25 & -0.8433 & -0.9253 & -0.9240 & -0.9337 & -0.9259 & -0.9251 \\
\hline 40.0 & 0.2 & 0.50 & -0.7326 & -0.8299 & -0.8283 & -0.8379 & -0.8304 & -0.8295 \\
\hline 40.0 & 0.2 & 0.75 & -0.6764 & -0.7890 & -0.7896 & -0.7952 & -0.7894 & -0.7887 \\
\hline 40.0 & 0.2 & 1.00 & -0.6415 & -0.7696 & -0.7704 & -0.7752 & -0.7700 & -0.7695 \\
\hline 42.5 & 0.2 & 0.25 & -0.6589 & -0.6999 & -0.6975 & -0.6953 & -0.7001 & -0.6999 \\
\hline 42.5 & 0.2 & 0.50 & -0.5902 & -0.6453 & -0.6445 & -0.6390 & -0.6455 & -0.6455 \\
\hline 42.5 & 0.2 & 0.75 & -0.5638 & -0.6323 & -0.6328 & -0.6239 & -0.6324 & -0.6325 \\
\hline 42.5 & 0.2 & 1.00 & -0.5472 & -0.6290 & -0.6285 & -0.6187 & -0.6290 & -0.6291 \\
\hline 45.0 & 0.2 & 0.25 & -0.4472 & -0.4657 & -0.4660 & -0.4664 & -0.4656 & -0.4657 \\
\hline 45.0 & 0.2 & 0.50 & -0.4584 & -0.4893 & -0.4897 & -0.4874 & -0.4891 & -0.4895 \\
\hline 45.0 & 0.2 & 0.75 & -0.4653 & -0.5080 & -0.5086 & -0.5022 & -0.5077 & -0.5082 \\
\hline 45.0 & 0.2 & 1.00 & -0.4654 & -0.5197 & -0.5198 & -0.5094 & -0.5194 & -0.5198 \\
\hline 47.5 & 0.2 & 0.25 & -0.2840 & -0.2921 & -0.2933 & -0.2919 & -0.2918 & -0.2922 \\
\hline 47.5 & 0.2 & 0.50 & -0.3607 & -0.3792 & -0.3801 & -0.3769 & -0.3788 & -0.3794 \\
\hline 47.5 & 0.2 & 0.75 & -0.3897 & -0.4186 & -0.4191 & -0.4130 & -0.4182 & -0.4187 \\
\hline 47.5 & 0.2 & 1.00 & -0.3999 & -0.4390 & -0.4393 & -0.4293 & -0.4386 & -0.4391 \\
\hline 40.0 & 0.3 & 0.25 & -0.7503 & -0.7828 & -0.7809 & -0.7794 & -0.7830 & -0.7828 \\
\hline 40.0 & 0.3 & 0.50 & -0.6915 & -0.7346 & -0.7337 & -0.7291 & -0.7347 & -0.7347 \\
\hline 40.0 & 0.3 & 0.75 & -0.6707 & -0.7247 & -0.7242 & -0.7172 & -0.7247 & -0.7248 \\
\hline 40.0 & 0.3 & 1.00 & -0.6570 & -0.7223 & -0.7221 & -0.7130 & -0.7223 & -0.7224 \\
\hline 42.5 & 0.3 & 0.25 & -0.6253 & -0.6451 & -0.6447 & -0.6440 & -0.6451 & -0.6451 \\
\hline 42.5 & 0.3 & 0.50 & -0.6152 & -0.6449 & -0.6451 & -0.6411 & -0.6448 & -0.6451 \\
\hline 42.5 & 0.3 & 0.75 & -0.6136 & -0.6534 & -0.6533 & -0.6459 & -0.6532 & -0.6535 \\
\hline 42.5 & 0.3 & 1.00 & -0.6091 & -0.6592 & -0.6593 & -0.6478 & -0.6590 & -0.6593 \\
\hline 45.0 & 0.3 & 0.25 & -0.5113 & -0.5233 & -0.5239 & -0.5234 & -0.5231 & -0.5233 \\
\hline 45.0 & 0.3 & 0.50 & -0.5510 & -0.5725 & -0.5728 & -0.5699 & -0.5723 & -0.5726 \\
\hline 45.0 & 0.3 & 0.75 & -0.5651 & -0.5959 & -0.5961 & -0.5893 & -0.5956 & -0.5960 \\
\hline 45.0 & 0.3 & 1.00 & -0.5674 & -0.6078 & -0.6080 & -0.5967 & -0.6075 & -0.6079 \\
\hline 47.5 & 0.3 & 0.25 & -0.4297 & -0.4377 & -0.4387 & -0.4364 & -0.4374 & -0.4377 \\
\hline 47.5 & 0.3 & 0.50 & -0.5027 & -0.5196 & -0.5206 & -0.5155 & -0.5192 & -0.5196 \\
\hline 47.5 & 0.3 & 0.75 & -0.5258 & -0.5515 & -0.5518 & -0.5437 & -0.5511 & -0.5515 \\
\hline 47.5 & 0.3 & 1.00 & -0.5320 & -0.5666 & -0.5668 & -0.5545 & -0.5662 & -0.5666 \\
\hline 40.0 & 0.4 & 0.25 & -0.7184 & -0.7372 & -0.7363 & -0.7353 & -0.7373 & -0.7373 \\
\hline 40.0 & 0.4 & 0.50 & -0.6996 & -0.7270 & -0.7270 & -0.7226 & -0.7270 & -0.7271 \\
\hline 40.0 & 0.4 & 0.75 & -0.6937 & -0.7303 & -0.7307 & -0.7227 & -0.7302 & -0.7304 \\
\hline 40.0 & 0.4 & 1.00 & -0.6871 & -0.7331 & -0.7332 & -0.7224 & -0.7330 & -0.7332 \\
\hline 42.5 & 0.4 & 0.25 & -0.6396 & -0.6526 & -0.6530 & -0.6523 & -0.6525 & -0.6527 \\
\hline 42.5 & 0.4 & 0.50 & -0.6553 & -0.6766 & -0.6769 & -0.6734 & -0.6765 & -0.6767 \\
\hline 42.5 & 0.4 & 0.75 & -0.6604 & -0.6902 & -0.6907 & -0.6832 & -0.6900 & -0.6903 \\
\hline 42.5 & 0.4 & 1.00 & -0.6585 & -0.6972 & -0.6976 & -0.6860 & -0.6970 & -0.6973 \\
\hline 45.0 & 0.4 & 0.25 & -0.5746 & -0.5839 & -0.5843 & -0.5833 & -0.5837 & -0.5840 \\
\hline 45.0 & 0.4 & 0.50 & -0.6190 & -0.6364 & -0.6367 & -0.6330 & -0.6361 & -0.6364 \\
\hline 45.0 & 0.4 & 0.75 & -0.6319 & -0.6574 & -0.6576 & -0.6502 & -0.6571 & -0.6574 \\
\hline 45.0 & 0.4 & 1.00 & -0.6333 & -0.6672 & -0.6673 & -0.6556 & -0.6669 & -0.6673 \\
\hline 47.5 & 0.4 & 0.25 & -0.5291 & -0.5365 & -0.5370 & -0.5347 & -0.5362 & -0.5365 \\
\hline 47.5 & 0.4 & 0.50 & -0.5908 & -0.6059 & -0.6064 & -0.6012 & -0.6056 & -0.6059 \\
\hline 47.5 & 0.4 & 0.75 & -0.6080 & -0.6309 & -0.6312 & -0.6226 & -0.6306 & -0.6310 \\
\hline 47.5 & 0.4 & 1.00 & -0.6113 & -0.6422 & -0.6423 & -0.6295 & -0.6419 & -0.6423 \\
\hline
\end{tabular}


(b) Option Deltas for Contract Set II

\begin{tabular}{|c|c|c|c|c|c|c|c|c|}
\hline (1) & $(2)$ & $(3)$ & $(4)$ & $(5)$ & $(6)$ & $(7)$ & $(8)$ & $(9)$ \\
\hline$S_{t}$ & $\sigma$ & $T-t$ & $\begin{array}{c}\text { European } \\
\text { price }\end{array}$ & $\begin{array}{c}\text { Bench- } \\
\text { mark }\end{array}$ & Ritchken & $\begin{array}{c}\text { Step-Func } \\
\text { w/ RE }\end{array}$ & $\begin{array}{c}\text { 3-Step MPE } \\
\text { w/o RE }\end{array}$ & $\begin{array}{c}\text { 3-Step MPE } \\
\text { w/ RE }\end{array}$ \\
\hline 49.5 & 0.2 & 0.25 & -0.2192 & -0.2244 & -0.2251 & -0.2225 & -0.2240 & -0.2245 \\
\hline 49.5 & 0.2 & 0.50 & -0.3126 & -0.3270 & -0.3273 & -0.3218 & -0.3266 & -0.3271 \\
\hline 49.5 & 0.2 & 0.75 & -0.3465 & -0.3703 & -0.3704 & -0.3616 & -0.3699 & -0.3704 \\
\hline 49.5 & 0.2 & 1.00 & -0.3590 & -0.3920 & -0.3921 & -0.3796 & -0.3916 & -0.3920 \\
\hline 49.5 & 0.3 & 0.25 & -0.3940 & -0.4006 & -0.4009 & -0.3979 & -0.4003 & -0.4007 \\
\hline 49.5 & 0.3 & 0.50 & -0.4755 & -0.4907 & -0.4908 & -0.4845 & -0.4903 & -0.4907 \\
\hline 49.5 & 0.3 & 0.75 & -0.5006 & -0.5242 & -0.5242 & -0.5143 & -0.5238 & -0.5242 \\
\hline 49.5 & 0.3 & 1.00 & -0.5078 & -0.5398 & -0.5398 & -0.5258 & -0.5395 & -0.5399 \\
\hline 49.5 & 0.4 & 0.25 & -0.5076 & -0.5144 & -0.5145 & -0.5114 & -0.5140 & -0.5144 \\
\hline 49.5 & 0.4 & 0.50 & -0.5737 & -0.5879 & -0.5879 & -0.5817 & -0.5876 & -0.5879 \\
\hline 49.5 & 0.4 & 0.75 & -0.5919 & -0.6137 & -0.6137 & -0.6038 & -0.6134 & -0.6138 \\
\hline 49.5 & 0.4 & 1.00 & -0.5956 & -0.6253 & -0.6253 & -0.6111 & -0.6250 & -0.6253 \\
\hline
\end{tabular}

Table 2 reports the delta values of American "up-and-out" put options on non-dividend-paying stocks for two sets of contracts computed using different methods. Set I includes 48 contracts, each of which has a different value of the parameter set $\left(S_{t}, T-t, \sigma\right)$. The domain of this parameter set is $S_{t}=(40,42.5,45,47.5)$, $T-t=(0.25,0.5,0.75,1.0)$, and $\sigma=(0.2,0.3,0.4)$. Set II is similar to set I, except that those contracts with $S_{t}=47.5$ are replaced by contracts with $S_{t}=49.5$. Panels (a) and (b) show the numerical results for contract set I and II, respectively. Columns 1 through 3 represent the values of the parameters, $S_{t}$ (the time- $t$ stock price), $\sigma$ (volatility), and $T-t$ (the time to expiration), respectively. Column 4 reports the European delta values obtained using the analytic formula in (15). Columns 5 and 6 show the numerical results of delta values from the Ritchken method with at least 10,000 time steps (the benchmark), and the Ritchken method with at least 50 time steps, respectively. Columns 7 through 9 show the numerical results from three analytic approximation techniques: the step-function scheme using a 4-point Richardson extrapolation, and the 3-step multi-piece exponential (MPE) approximation with and without Richardson extrapolation. 
Table 3: Summary of Results on Option Prices and Delta Values of American "Up-and-Out" Put Options on Non-Dividend-Paying Stocks

$$
(K=\$ 45 ; H=\$ 50 ; r=4.88 \%)
$$

\begin{tabular}{|c|c|c|c|c|}
\hline \multirow[t]{3}{*}{ (1) } & $(2)$ & $(3)$ & $(4)$ & $(5)$ \\
\hline & \multicolumn{2}{|c|}{ Contract Set I } & \multicolumn{2}{|c|}{ Contract Set II } \\
\hline & Price & Delta & Price & Delta \\
\hline & \multicolumn{4}{|c|}{ Ritchken } \\
\hline RMSE & $3.349 \mathrm{e}-03$ & $7.190 \mathrm{e}-04$ & $3.278 \mathrm{e}-03$ & $6.600 \mathrm{e}-04$ \\
\hline RMSRE & $1.254 \mathrm{e}-03$ & $1.233 \mathrm{e}-03$ & $1.096 \mathrm{e}-03$ & $1.022 \mathrm{e}-03$ \\
\hline \multirow[t]{2}{*}{ CPU Time (sec) } & $5.300 \mathrm{e}-01$ & $5.300 \mathrm{e}-01$ & $7.416 \mathrm{e}+01$ & $7.416 \mathrm{e}+01$ \\
\hline & \multicolumn{4}{|c|}{ Cheuk-Vorst } \\
\hline RMSE & $2.408 \mathrm{e}-03$ & NA & $2.195 \mathrm{e}-03$ & NA \\
\hline RMSRE & $1.258 \mathrm{e}-03$ & NA & $2.535 \mathrm{e}-03$ & NA \\
\hline \multirow[t]{2}{*}{ CPU Time (sec) } & $7.500 \mathrm{e}-01$ & NA & $7.500 \mathrm{e}-01$ & NA \\
\hline & \multicolumn{4}{|c|}{ Step-Func w/RE } \\
\hline RMSE & $5.178 \mathrm{e}-03$ & $6.975 \mathrm{e}-03$ & $4.836 \mathrm{e}-03$ & $7.464 \mathrm{e}-03$ \\
\hline RMSRE & $2.132 \mathrm{e}-03$ & $1.115 \mathrm{e}-02$ & $2.231 \mathrm{e}-03$ & $1.287 \mathrm{e}-02$ \\
\hline \multirow[t]{2}{*}{ CPU Time (sec) } & $1.600 \mathrm{e}-01$ & $1.600 \mathrm{e}-01$ & $1.600 \mathrm{e}-01$ & $1.600 \mathrm{e}-01$ \\
\hline & \multicolumn{4}{|c|}{ 3-Step MPE w/o RE } \\
\hline RMSE & $1.733 \mathrm{e}-03$ & $2.727 \mathrm{e}-04$ & $1.673 \mathrm{e}-03$ & $2.797 \mathrm{e}-04$ \\
\hline RMSRE & $6.518 \mathrm{e}-04$ & $4.881 \mathrm{e}-04$ & $7.014 \mathrm{e}-04$ & $5.670 \mathrm{e}-04$ \\
\hline \multirow[t]{2}{*}{ CPU Time (sec) } & $3.000 \mathrm{e}-01$ & $3.000 \mathrm{e}-01$ & $3.000 \mathrm{e}-01$ & $3.000 \mathrm{e}-01$ \\
\hline & \multicolumn{4}{|c|}{ 3-Step MPE w/RE } \\
\hline RMSE & $5.991 \mathrm{e}-04$ & $1.261 \mathrm{e}-04$ & $5.937 \mathrm{e}-04$ & $1.242 \mathrm{e}-04$ \\
\hline RMSRE & $1.786 \mathrm{e}-04$ & $2.047 \mathrm{e}-04$ & $1.728 \mathrm{e}-04$ & $2.011 \mathrm{e}-04$ \\
\hline CPU Time (sec) & $5.700 \mathrm{e}-01$ & $5.700 \mathrm{e}-01$ & $5.700 \mathrm{e}-01$ & $5.700 \mathrm{e}-01$ \\
\hline
\end{tabular}

Table 3 reports a summary of the results from the Ritchken, the Cheuk-Vorst, and the three analytical approximation methods for American "up-and-out" put options on non-dividend-paying stocks for two sets of contracts. Set I includes 48 contracts, each of which has a different value of the parameter set $\left(S_{t}, T-t, \sigma\right)$. The domain of this parameter set is $S_{t}=(40,42.5,45,47.5), T-t=(0.25,0.5,0.75,1.0)$, and $\sigma=(0.2,0.3,0.4)$. Set II is similar to set I, except that those contracts with $S_{t}=47.5$ are replaced by contracts with $S_{t}=49.5$. Columns 2 and 3 show the numerical results of option prices and delta values for contract set I. Columns 4 and 5 show the numerical results of option prices and delta values for contract set II. Beginning with row 3, deviation from the benchmark - the results from the Ritchken method with at least 10,000 time steps - is reported for each of the following five methods: the Ritchken method with at least 50 time steps, the Cheuk and Vorst with 100 time steps, the step-function scheme using a 4-point Richardson extrapolation, and the 3-step multi-piece exponential (MPE) approximation with and without Richardson extrapolation.

The root of the mean squared error (RMSE) and the root of the mean squared relative error (RMSRE) are used as two measures of deviation from the benchmark. The CPU time is the amount of the time required for each method on a Sun Ultra 1 workstation to compute the option prices or delta values for all the 48 contracts in a given set of contracts. 
Table 4: Summary of Results on Option Prices and Delta Values of Low- and High-Premium American "Up-and-Out" Put Options

$$
(K=\$ 45 ; H=\$ 50 ; r=4.88 \%)
$$

(a) Low Premium Contracts

\begin{tabular}{|c|c|c|c|c|}
\hline \multirow[t]{3}{*}{$(1)$} & (2) & (3) & (4) & (5) \\
\hline & \multicolumn{2}{|c|}{ Contract Set I } & \multicolumn{2}{|c|}{ Contract Set II } \\
\hline & Price & Delta & Price & Delta \\
\hline & \multicolumn{4}{|c|}{ Step-Func w/RE } \\
\hline RMSE & 4.409E-03 & $5.479 \mathrm{E}-03$ & $3.548 \mathrm{E}-03$ & $6.645 \mathrm{E}-03$ \\
\hline \multirow[t]{2}{*}{ RMSRE } & $2.617 \mathrm{E}-03$ & $9.830 \mathrm{E}-03$ & $2.776 \mathrm{E}-03$ & $1.340 \mathrm{E}-02$ \\
\hline & \multicolumn{4}{|c|}{ 3-Step MPE w/o RE } \\
\hline RMSE & $1.371 \mathrm{E}-03$ & $2.849 \mathrm{E}-04$ & $1.213 \mathrm{E}-03$ & $2.981 \mathrm{E}-04$ \\
\hline \multirow[t]{2}{*}{ RMSRE } & $7.704 \mathrm{E}-04$ & $5.869 \mathrm{E}-04$ & $8.531 \mathrm{E}-04$ & 7.149E-04 \\
\hline & \multicolumn{4}{|c|}{ 3-Step MPE w/RE } \\
\hline RMSE & $3.179 \mathrm{E}-04$ & $8.693 \mathrm{E}-05$ & $2.974 \mathrm{E}-04$ & $8.034 \mathrm{E}-05$ \\
\hline RMSRE & $1.729 \mathrm{E}-04$ & $1.831 \mathrm{E}-04$ & $1.606 \mathrm{E}-04$ & $1.750 \mathrm{E}-04$ \\
\hline
\end{tabular}

(b) High Premium Contracts

\begin{tabular}{c|cccc}
\hline \hline$(1)$ & $(2)$ & $(3)$ & $(4)$ & $(5)$ \\
& Contract & Set I & \multicolumn{1}{c}{ Contract Set II } \\
\hline & \multicolumn{4}{|c}{ Step-Func w/RE } \\
RMSE & $5.846 \mathrm{E}-03$ & $8.202 \mathrm{E}-03$ & $5.846 \mathrm{E}-03$ & $8.202 \mathrm{E}-03$ \\
RMSRE & $1.498 \mathrm{E}-03$ & $1.233 \mathrm{E}-02$ & $1.498 \mathrm{E}-03$ & $1.233 \mathrm{E}-02$ \\
& & 3-Step MPE w/o RE & \\
\cline { 3 - 5 } RMSE & $2.031 \mathrm{E}-03$ & $2.599 \mathrm{E}-04$ & $2.031 \mathrm{E}-03$ & $2.599 \mathrm{E}-04$ \\
RMSRE & $5.061 \mathrm{E}-04$ & $3.633 \mathrm{E}-04$ & $5.061 \mathrm{E}-04$ & $3.633 \mathrm{E}-04$ \\
& & 3-Step MPE w/RE & \\
RMSE & $7.852 \mathrm{E}-04$ & 1.562E-04 & $7.852 \mathrm{E}-04$ & $1.562 \mathrm{E}-04$ \\
RMSRE & $1.842 \mathrm{E}-04$ & $2.241 \mathrm{E}-04$ & $1.842 \mathrm{E}-04$ & $2.241 \mathrm{E}-04$ \\
\hline
\end{tabular}

Table 4 reports a summary of the results from the analytic methods for both low- and high-premium out-ofthe-money American "up-and-out" put options on non-dividend-paying stocks for two sets of contracts. Set I includes 48 contracts, each of which has a different value of the parameter set $\left(S_{t}, T-t, \sigma\right)$. The domain of this parameter set is $S_{t}=(40,42.5,45,47.5), T-t=(0.25,0.5,0.75,1.0)$, and $\sigma=(0.2,0.3,0.4)$. Set II is similar to set I, except that those contracts with $S_{t}=47.5$ are replaced by contracts with $S_{t}=49.5$. Panels (a) and (b) summarize, respectively, the results for low- and high-premium options within each set of contracts. High-premium contracts within each set are those whose premium (the difference between the benchmark American and European option values) are ranked in the top half of the group, whereas low-premium contracts within each set are those whose premium ranked in the bottom half. Columns 2 and 3 show the numerical results of option prices and delta values for contract set I. Columns 4 and 5 show the numerical results of option prices and delta values for contract set II. Within each panel, deviation from the benchmark - the results from the Ritchken method with at least 10,000 time steps - is reported for each of the following three methods: the step-function scheme using a 4-point Richardson extrapolation, and the 3-step multi-piece exponential (MPE) approximation with and without Richardson extrapolation. The root of the mean squared error (RMSE) and the root of the mean squared relative error (RMSRE) are used as two measures of deviation from the benchmark. 


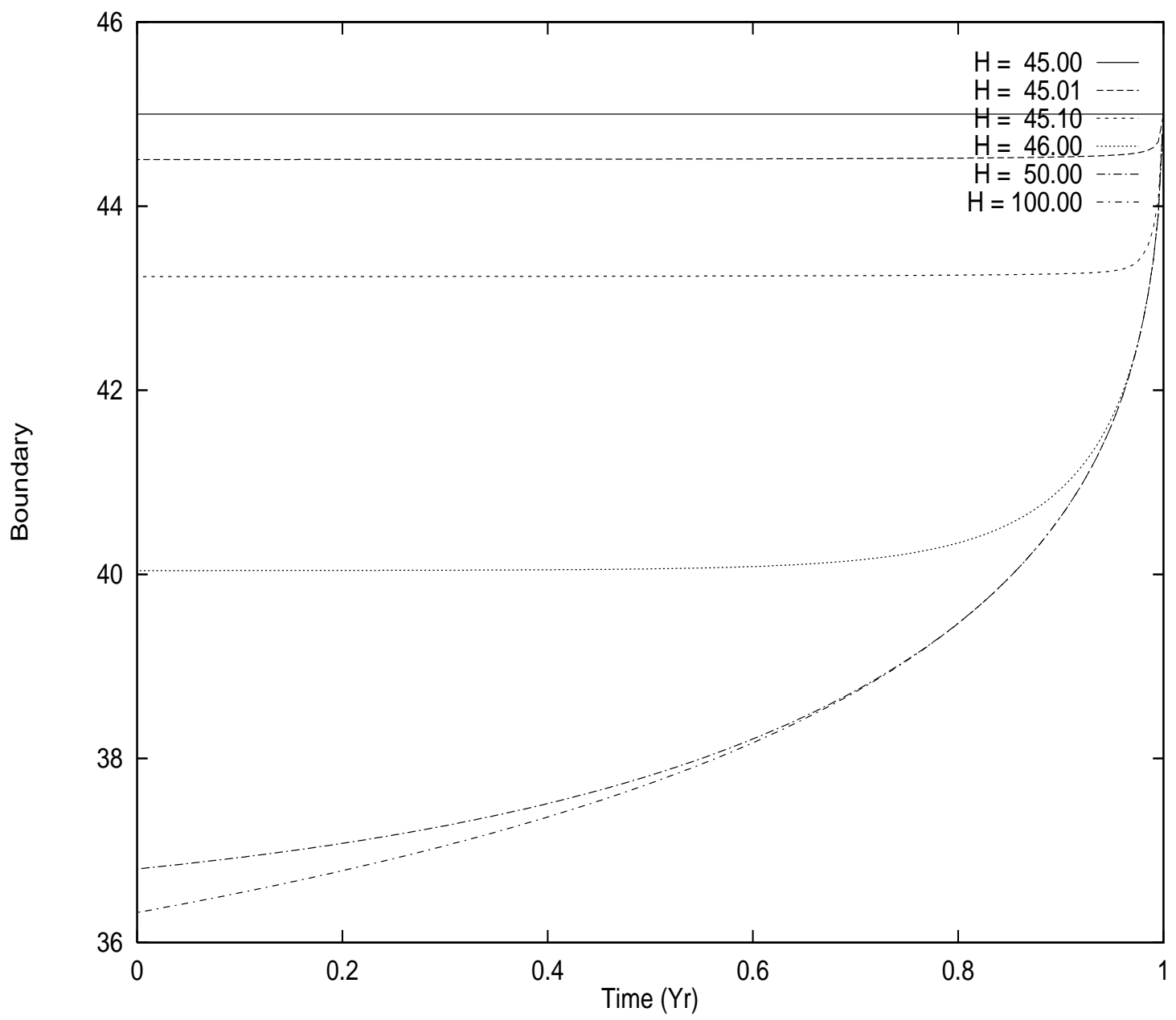

Figure 1: Optimal Exercise Boundaries of American Barrier Options with Different Barrier Levels

Figure 1 shows the plots of the optimal exercise boundary for American out-of-the-money "up-andout" put options on non-dividend-paying stocks for different values of the barrier level $H$, namely $H=45,45.01,45.10,46,50,100$. The values of other relevant parameters are: strike price $K=45$, time to expiration $T-t=1$ (year), volatility $\sigma=0.2$, and risk-free rate $r=0.0488$. The boundary with $H=45$ coincides with the strike price. The boundary with $H=100$ is essentially the same as the boundary of an otherwise identical standard American option $(H=\infty)$. Each boundary shown here is generated using 200 points (time-steps). 


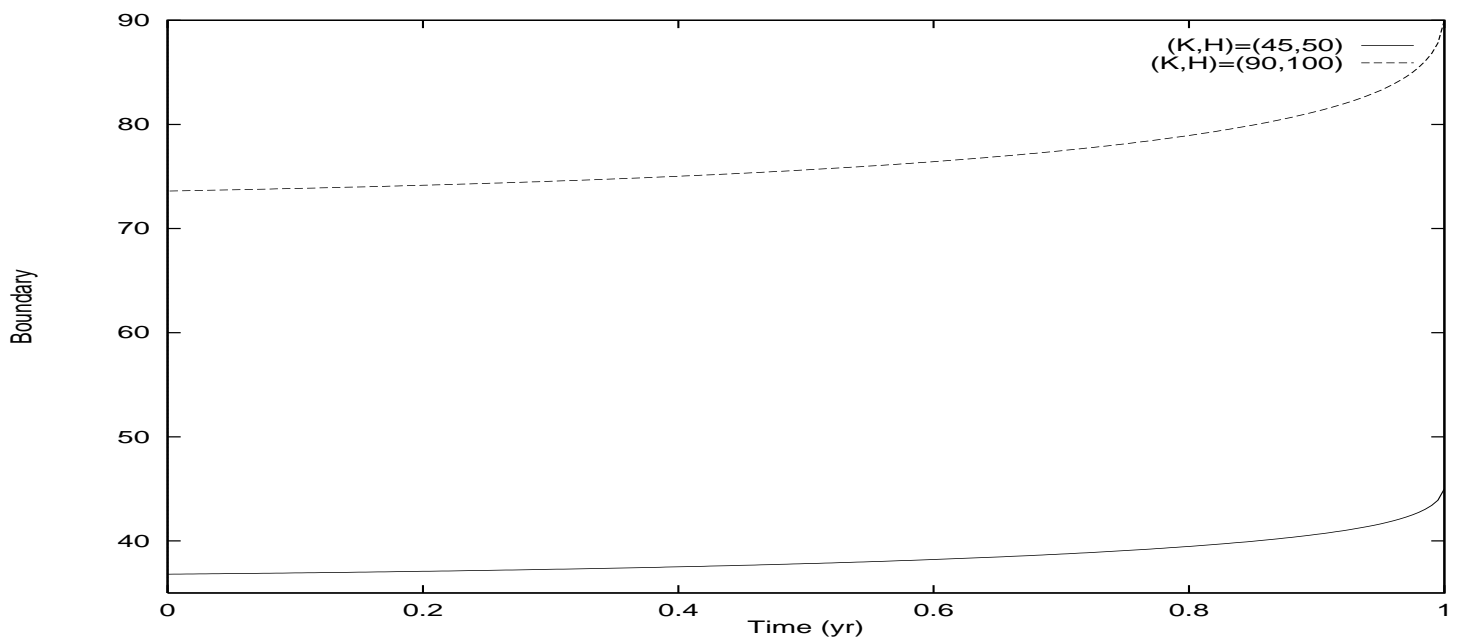

(a)

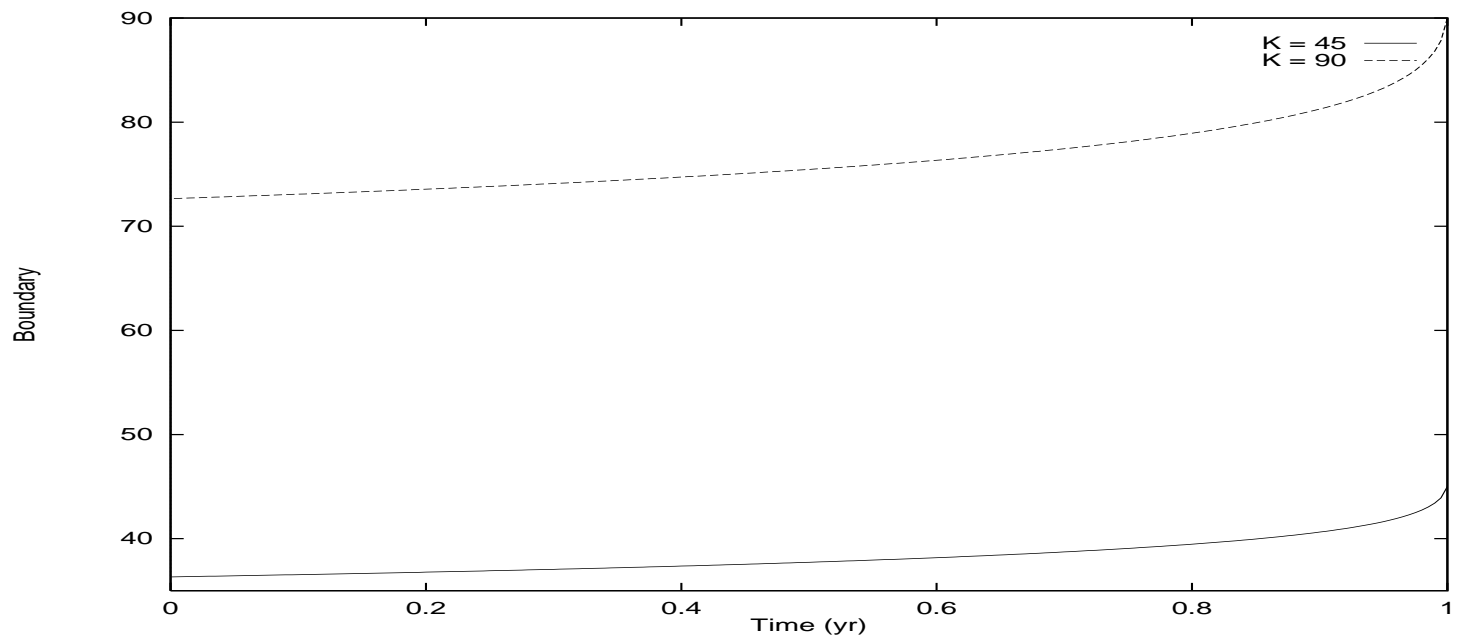

(b)

Figure 2: Price Homogeneity of Optimal Exercise Boundaries of American "Up-and-Out" Put Options

Figure 2 illustrates the price homogeneity of the optimal exercise boundary for American out-of-the-money "up-and-out" put options on non-dividend-paying stocks. Figure 2(a) shows plots of the boundary with $(K=45, H=50)$ and $(K=90, H=100)$ to illustrate the homogeneity in $(K, H)$. Figure 2(b) shows plots of the boundary with $(K=45, H=100)$ and $(K=90, H=500)$ to illustrate the homogeneity in $K$ when $H \gg K$ or essentially when $H=\infty$. In both (a) and (b), the height of the dashed curve is twice the height of the solid curve (homogeneity). The values of other relevant parameters are time to expiration $T-t=1$ (year), volatility $\sigma=0.2$, and risk-free rate $r=0.0488$. Each boundary shown here is generated using 200 points (time-steps). 


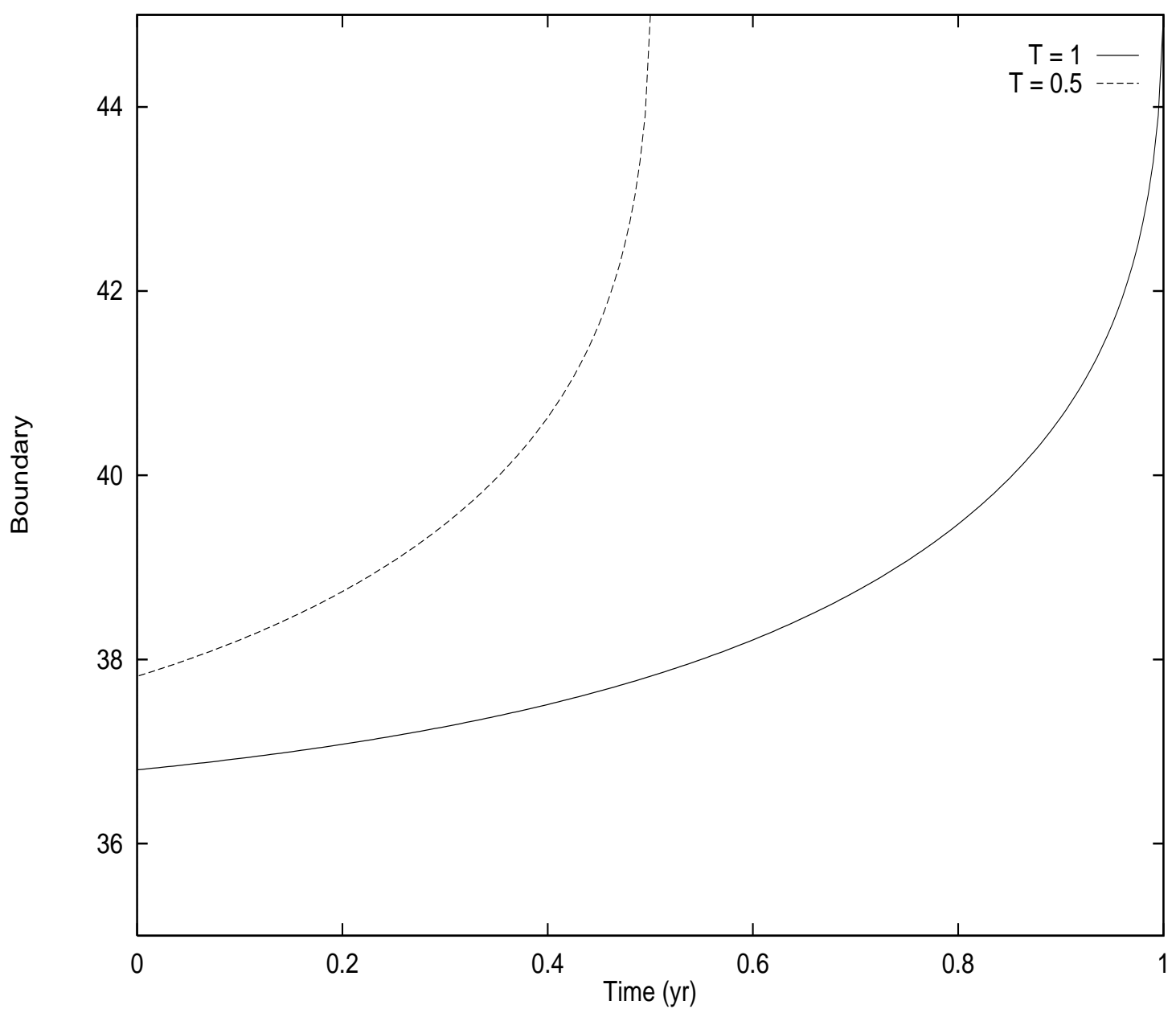

Figure 3: Stationarity of Optimal Exercise Boundaries of American "Up-and-Out" Put Options

Figure 3 illustrates the stationarity of the optimal exercise boundary of American out-of-the-money "upand-out" put options on non-dividend-paying stocks. Two plots of the boundary are shown in the figure and differ only in time to expiration, one with $T-t=0.5$ (year) and the other with $T-t=1$ (year). When shifted to the right for $T-t=0.5$, the dashed curve will coincide with the solid curve (stationarity). The values of other relevant parameters are strike $K=45$, barrier $H=50$, volatility $\sigma=0.2$, and risk-free rate $r=0.0488$. Each boundary shown here is generated using 200 points (time-steps). 